\title{
A Global Search Framework for Practical Three-Dimensional Packing with Variable Carton Orientations
}

\author{
Yaohua He ${ }^{\text {a, } \mathrm{c}^{*}}$, Yong Wu ${ }^{\text {b }}$, Robert de Souza ${ }^{\mathrm{c}}$ \\ a Dept. of Industrial and Systems Engineering / Office of the Provost (PVO), National University of Singapore, Singapore 117576 \\ b Department of International Business and Asian Studies, Gold Coast Campus, Griffith University, QLD 4222 Australia \\ c The Logistics Institute - Asia Pacific, National University of Singapore, Singapore 119613
}

\begin{abstract}
This article aims to tackle a practical three-dimensional packing problem, where a number of cartons of diverse sizes are to be packed into a bin with fixed length and width but open height. Each carton is allowed to be packed in any one of the six orientations, and the carton edges are parallel to the bin edges. The allowance of variable carton orientations exponentially increases the solution space and makes the problem very challenging to solve. This study first elaborately devises the packing procedure which converts an arbitrary sequence of cartons into a compact packing solution and subsequently develops an improved genetic algorithm (IGA) to evolve a set of solutions. Moreover, a novel global search framework (GSF), utilizing the concept of evolutionary gradient, is proposed to further improve the solution quality. Numerical experiments indicate that IGA provides faster and better results and GSF demonstrates its superior performance, especially in solving relative large-size and heterogeneous instances. Applying the proposed algorithms to some benchmarking cases of the three-dimensional strip packing problem also indicates that the algorithms are robust and effective compared to existing methods in the literature.
\end{abstract}

Keywords: packing; loading; three-dimensional; genetic algorithm; global search; evolutionary gradient

\section{Introduction}

Bin packing, or container/pallet loading, is a very common but important task in the manufacturing and logistics industries. However, even after the problem has been researched for several decades, many of packing tasks are still accomplished manually, i.e., the workers stow bins or containers according to their experience and judgement. It is evident from operational observations and the literature that manual packing may not optimally utilize the valuable bin/container space, and the volume utilization can be significantly improved with the support of computer-generated solutions. Exact algorithms, due to the complexity and combinatorial explosive nature, can only solve small-size problems to optimality with the current computing technology. For most practical applications, heuristics and meta-heuristics are still the best choices to achieve optimal or near-optimal solutions within reasonable time.

This study solves a practical three-dimensional packing problem by using heuristics and meta-heuristics with the purpose of obtaining high quality solutions as fast as possible. In the investigated problem, a number

\footnotetext{
* Corresponding Author: Tel: +65-90438961, Fax: +65-67771434

E-mail: Yaohua.He001@gmail.com (Y. He),yong.wu@griffith.edu.au, wuyong@pmail.ntu.edu.sg (Y. Wu)
} 
of cartons of diverse sizes are to be stowed into a paperboard bin with fixed length and width but open height (subject to physical limit), i.e., the bin height can be trimmed to fit the content of the bin to save transport cost (e.g., when air transportation is used). Each carton is allowed to be packed in any one of the six orientations, and the carton edges are parallel to the bin edges (we will call this as variable orientations hereafter). The cartons need not be stowed in layers or walls, and no so-called guillotine constraint is imposed to the packing process.

According to the typology of cutting and packing problems introduced by Wäscher et al. (2007), our investigated problem is a three-dimensional packing problem with one variable dimension, different from the traditional three-dimensional bin packing problem (3D-BPP) where a selected subset of a number of cartons are packed into a bin with fixed length, width and height to maximize the volume utilization, or where a large number of cartons are packed into identical bins with the objective to minimize the number of bins used. For this problem, the variable bin height poses a greater challenge in providing high-quality solutions since usually no selection of cartons is allowed, i.e., all cartons need to be packed, and when the bin is not fully packed the extra height will be trimmed. The flexibility of carton orientations further expands the solution space significantly and thus increases the difficulty in finding the optimal solutions. Up to now, such a problem with both variable bin height and variable carton orientations has only been studied by a few researchers.

It should be noted that the problem under investigation possesses some characteristics of the three-dimensional strip packing problem (3D-SPP). However, compare with the traditional 3D-SPP in the literature, it presents two important features: a) the physical bin height (which corresponds to strip length in 3D-SPP) limit is relatively short due to the fact that paperboard bins are used, which limits the maximum loading height/weight; whereas in 3D-SPP the strip length is usually much longer than both the strip width and the strip height, thus the term 'strip' is used; b) the cartons that are to be packed are 'generated' on the fly in various dimensions, which presents a very heterogeneous packing problem; in contrast in traditional 3D-SPP it is common to see boxes (in small bunches) have the same dimensions. The demand to pack highly heterogeneous cartons in a relatively small paperboard bin makes layer-building heuristics unsuitable (e.g., in extreme case, every carton to be packed may belong to a different carton type, this may make layer-building almost impossible). Although the current problem represents a special case of 3D-SPP, the concept of 'bin' will still be used for ease of description in this paper for two reasons: a) paperboard bins are used in practical packing; and b) there is no significant difference between the maximum (variable) bin height and the fixed bin length and fixed bin width.

The solution techniques for packing problems can be classified into three types:

Exact methods based on mathematical programming belong to the first type. Chen et al. (1995) provided a mixed integer linear programming (MILP) model to solve the 3D-BPP with variable orientations. Due to the strong NP-hardness of the problem, this MILP model only solved a small instance with 6 cartons to optimality. Martello et al. (2000) and den Boef et al. (2005) presented an exact algorithm for loading a single bin and discussed the lower bounds for the 3D-BPP. Martello et al. (2007) presented an algorithm to 
solve moderate instances to optimality for the general 3D-BPP and its robot-packable variant. Fekete et al. (2007) developed a two-level tree search algorithm for solving higher-dimensional packing problems to optimality, but their computational results reported were on the optimal solutions for two-dimensional test problems from the literature. Ceselli and Righini (2008) presented a branch-and-price algorithm for the exact optimization of the ordered open-end bin-packing problem defined by Yang and Leung (2003), where the items to be packed are sorted in a given order and the capacity of each bin can be exceeded by the last item packed into the bin.

Heuristics and approximate algorithms comprise the second type. Lim et al. (2005) conducted a comprehensive review on the heuristics studied by many researchers, from which it could be seen that most heuristics were on the wall- or layer-building of the traditional 3D-BPP to pack a selected subset of a number of cartons into a bin/container pursuing high volume utilization. For example, George and Robinson (1980) presented a wall-building approach heuristic without restrictions on the carton orientations for identical containers. Bischoff and Marroitt (1990) evaluated 14 different heuristics based on the George-Robinson framework. Bischoff et al. (1995) proposed a pallet loading heuristic for non-identical cartons where the stability of the pallet was considered. Pisinger (2002) offered a heuristic based on the wall-building approach, where strips and layers were created so that the 3D-BPP could be decomposed into smaller sub-problems. Baltacioglu et al. (2006) developed a new heuristic algorithm using rules to mimic human intelligence to solve the 3D-BPP. Faroe et al. (2003) provided a heuristic for packing cartons into a minimum number of identical containers based on guided local search, however, no variable orientation was allowed. Huang and He (2009) developed a heuristic algorithm for the traditional 3D-BPP without orientation constraints based on the concept of caving degree inspired by an old adage "Gold corner, silver side and grass belly" from Chinese Weiqi (a board game), which achieved very high volume utilization for the benchmark instances provided by Bischoff and Ratcliff (1995) and later solved by so many researchers, such as Lim et al. (2005), Bortfeldt and Gehring $(1998,2001)$ and Zhang et al. (2007).

The third choice is meta-heuristics, such as genetic algorithms, tabu search and simulated annealing, are widely used. Gehring and his colleagues have published a number of papers on meta-heuristic methods for 3D-BPP. Gehring and Bortfeldt (1997) first presented a genetic algorithm and then (2002) provided a parallel genetic algorithm for single container loading with stronger heterogeneous cartons. Bortfeldt and Gehring (1998) first applied tabu search and then (2001) presented a hybrid genetic algorithm for single container loading, finding that the former was suitable to weakly heterogeneous cases but the latter suitable to strongly heterogeneous cases. Lodi et al. (2002) provided a tabu search framework by exploiting a constructive heuristic to evaluate the neighborhood where the carton orientations were fixed. Zhang et al. (2007) proposed a simulated annealing algorithm combined with a bin-loading heuristic to solve the classic 3D-BPP. Crainic et al. (2008a) introduced a two-level tabu search where the first level aimed to reduce the number of bins and the second optimized the bin packing of cartons with fixed orientations. Egeblad and Pisinger (2009) examined 2D/3D knapsack packing problems using simulated annealing. Goncalvez and Resende (2012) proposed a parallel random-key-based genetic algorithm using layer-building heuristics. 
Another classification for 3D loading problems by Fanslau and Bortfeldt (2010) divides packing algorithms into exact and heuristic methods. The latter can be further divided into three groups: conventional heuristics, meta-heuristics and tree search methods. Bortfeldt and Mack (2007) presented a layer-building heuristic method for the 3D-SPP, where tree search algorithms were adopted to determine the best layer depths and to obtain the best strip directions and strip dimensions for a given layer. Their method did not involve any supplementary loading restrictions, particularly no supporting constraints are imposed. Regarding the heuristic packing approaches (Pisinger, 2002), wall building, stack building, horizontal layer building, block building and guillotine cutting were often used.

Allen et al. (2011) proposed a hybrid placement strategy for the 3D-SPP, which is actually a decomposition method: one bigger sub-set of items are packed via three-dimensional best fit heuristic, the other smaller sub-set of items are packed through a tabu search algorithm, thus saving the search time.

Through analyzing the existing solution methods, the following observations can be made: (1) Most of heuristics are based on the wall-building with some modifications, which are in nature greedy techniques that might stuck at local optimal solutions and away from the global optimality; (2) Exact methods are based on mathematical programming and graph theory by reducing the original problem to a theoretically solvable model, which at the moment would be virtually impossible to guarantee the global optimality for practical 3D problems, especially for those with variable orientations; (3) Meta-heuristics are the present best choice, which can search much larger feasible space than the simple heuristics and converge much faster than the exact methods, hence make it realistic to obtain optimal or near-optimal solutions within reasonable search time.

However, in practical applications of the meta-heuristics, the users usually have to tune the algorithm parameters according to the problem conditions and carry out a number of tests to select the best solution as the real stowage plan. In general, the users hope to have lower variance of solutions in the tests, which may imply the algorithm has higher search performance and convergence. But the search ability of an algorithm depends on the prevailing problem conditions. As long as the solution variance exists, all the better solutions via the algorithm are still possible. So how to fully utilize the search ability until the average deviation of solutions turns to be null will be considered in this paper.

This paper further studies the problem proposed in $\mathrm{Wu}$ et al. (2010) in which an exact mathematical model based on Chen et al. (1995) was first developed and a genetic algorithm was subsequently designed and implemented with a heuristic packing procedure (or decoding routine). In this paper, we first improve the decoding procedure aiming at tighter packing and higher computational efficiency; and then redesign and implement a genetic algorithm with well tailored components based on the work of $\mathrm{He}$ (2007 and 2009). Further, we propose a global search framework based on the concept of evolutionary gradient (He and Hui, 2009 and 2010) and devise several scenarios of the framework for simulation experiments. A suitable scenario is selected for the practical 3D packing problem through numerical experiments.

\section{Problem Description}


The investigated problem originates from a real-world application, for which $\mathrm{Wu}$ et al. (2010) provided an MILP model and a basic GA. The packing objective is to minimize the height $H$ of the paperboard bin used when packing $N$ cartons so that logistics costs can be saved since the packed bins are transported via air. As mentioned earlier, the height of bins is subject to physical limit.

\subsection{Bins and Cartons}

In the practical problem, a rectangular bin with fixed length and width $(L \times W)$ is available to pack a number $(N)$ of rectangular cartons for shipping (see Fig. 1a). The height $(H)$ of the bin can be trimmed to fit the cartons packed, but cannot exceed the physical limit height, $H_{\max }$.

Each carton $i$ has a fixed size of $l_{i} \times w_{i} \times h_{i}$ (centimeter), where $l_{i}, w_{i}$ and $h_{i}$ are the length, width and height of carton $i$. Without loss of generality, we assume $l_{i} \geq w_{i} \geq h_{i}$ (see Fig. 1b). Each carton may be packed into the bin in any one of the six orientations that keep the carton edges parallel to the bin edges. The cartons need not be stowed in layers or walls, and no so-called guillotine constraint is imposed to the packing process.

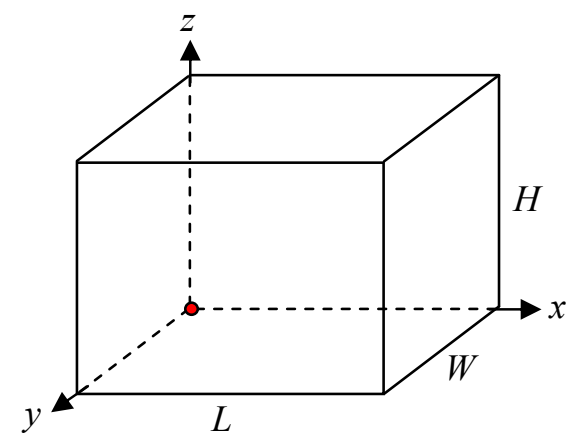

(a) A bin with $L \times W \times H$

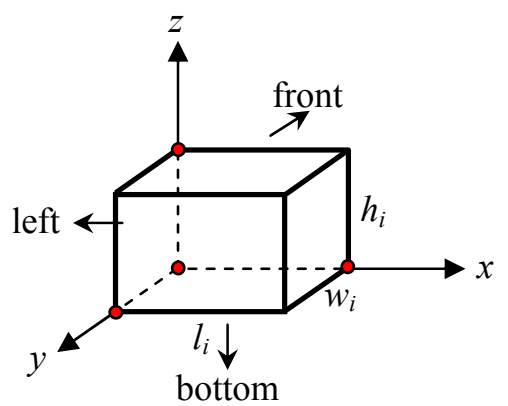

(b) A carton $i$ with $l_{i} \times w_{i} \times h_{i}$

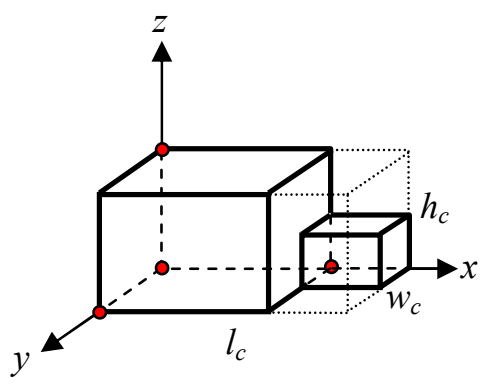

(c) A cage bin with $l_{c} \times w_{c} \times h_{c}$

Fig. 1 A bin, a carton and a cage bin

\subsection{Reference Points, Cage Bin and Orientations}

The Cartesian coordinate system is used to locate a carton in the bin (see Fig. 1a). Martello et al. (2000) proposed corner points as candidate locations where a carton could be placed, which needs be calculated by using a 2D algorithm and bears the shortcoming that some space of the bin is not covered and thus cannot be exploited. Zhang et al. (2007) presented possible placement points for a carton to be placed, a selected point of which is just a possible location for a carton, but may not be the final position because of the later parallel moves for compact packing. Therefore placement points belong to a kind of reference points. Crainic et al. (2008b) introduced extreme points and corresponding heuristics for 3D-BPP without any further parallel moving, even though extreme points are generated in the same way as placement points. We adopt the concept of reference points in this work.

When each carton $i$ is packed into the bin, the carton edges are parallel to the bin edges, as shown in Fig. 1(b). A reference point in the bin is denoted by its coordinates $(x, y, z)$. An empty bin has only one reference point $(0,0,0)$, and every time when the left-front-bottom corner of carton $i$ with $l_{i} \times w_{i} \times h_{i}$ is assigned at $\left(x_{i}, y_{i}\right.$, $\left.z_{i}\right)$, three new reference points will be generated depending on the carton orientation, such as $\left(x_{i}+l_{i}, y_{i}, z_{i}\right),\left(x_{i}\right.$, 
$\left.y_{i}+w_{i}, z_{i}\right)$ and $\left(x_{i}, y_{i}, z_{i}+h_{i}\right)$.

The concept of cage bin is utilized in our work. A cage bin is a virtual bin and is defined as the smallest rectangular box (with dimensions of $l_{c} \times w_{c} \times h_{c}$ ) that is able to encase all the cartons already packed in the bin; therefore, the cage bin changes as the packing of cartons proceeds. For example, Fig. 1c shows the cage bin encasing two cartons packed so far in the bin.

Each carton can be placed into the bin in any one of its six orientations. That is to say, although the original size $\left(l_{i} \times w_{i} \times h_{i}\right)$ of carton $i$ is fixed, its orientation in the bin is not pre-fixed. To make the total packing compact, each carton $i$ can take any one of its six orientations as depicted in Fig. 2, where $\gamma_{i}$ denote the orientation number, $\gamma_{i} \in\{1,2,3, \ldots, 6\}, i=1,2,3, \ldots, N$.
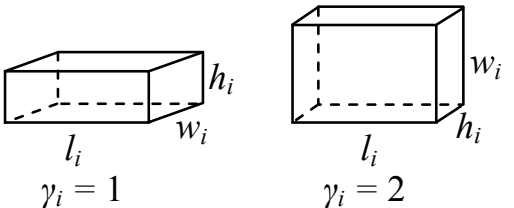

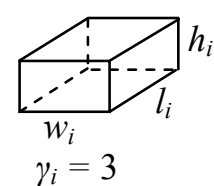

$\gamma_{i}=3$

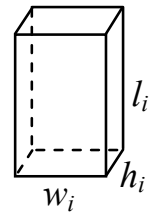

$\gamma_{i}=4$
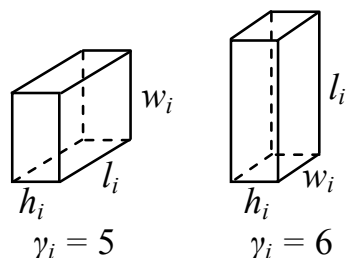

Fig. 2 Six orientations of carton $i$

\subsection{An MILP Solvable Example}

Mathematically proven global optimal solutions, no matter how difficult to derive, are always desirable for first of all, providing a benchmark for other methods; and secondly, applying to the actual problem when the solutions are relatively easy to achieve. Wu et al. (2010) adopted the MILP model presented in Chen et al. (1995) and coded in GAMS. Though computing resources have advanced significantly in the past two decades, the mathematical approach still seems to be less practical, as shown by the following example.

Table 1 presents the original sizes of a set of cartons to be packed into a small bin with fixed length and width $L \times W=80 \times 58 \mathrm{~cm}$, the $H_{\max }=95 \mathrm{~cm}$. This example (called SM00) will be used to illustrate the proposed solution techniques in this paper.

Table 1. The sizes of the cartons in SMOO

\begin{tabular}{|c|c|c|c|}
\hline Carton $\boldsymbol{i}$ & $\boldsymbol{l}_{\boldsymbol{i}} \times \boldsymbol{w}_{\boldsymbol{i}} \times \boldsymbol{h}_{\boldsymbol{i}}$ & Carton $\boldsymbol{i}$ & $\boldsymbol{l}_{\boldsymbol{i}} \times \boldsymbol{w}_{\boldsymbol{i}} \times \boldsymbol{h}_{\boldsymbol{i}}$ \\
\hline 1 & $36 \times 36 \times 36$ & 6 & $40 \times 31 \times 21$ \\
\hline 2 & $59 \times 39 \times 20$ & 7 & $31 \times 31 \times 17$ \\
\hline 3 & $54 \times 40 \times 21$ & 8 & $31 \times 17 \times 16$ \\
\hline 4 & $58 \times 37 \times 21$ & 9 & $26 \times 23 \times 14$ \\
\hline 5 & $52 \times 33 \times 20$ & 10 & $33 \times 21 \times 4$ \\
\hline
\end{tabular}

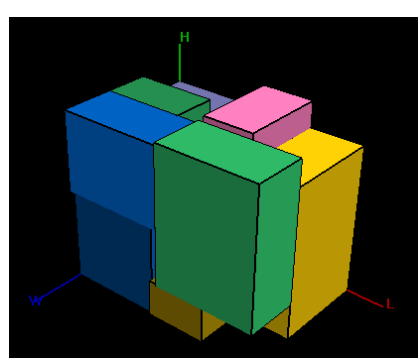

Fig. 3 Optimal packing for SM00

The above example was solved by the commercial solver CPLEX 11.2 based on the GAMS model in Wu et al. (2010). CPLEX 11.2 finds the solution with the height $68 \mathrm{~cm}$ after 1006 seconds, and proves the optimality at 1144 second. Fig. 3 shows the packing configuration of the optimal solution.

It can be observed from the example that mathematical approach still takes a long time to solve a relatively small practical instance. An optimistic estimation of the maximum number of cartons the mathematical model can handle under current computational resources would be below 20, or even 15 . In 
Wu et al. (2010), such a maximum number was 12 for CPLEX 11.0 with 2 hours' running time and in a number of cases where less than 12 cartons were presented, CPLEX 11.0 failed to find the proven optimal solutions within the specified running time. Therefore, to provide solutions for practical problems within reasonable time, alternative approaches must be used.

\section{The GA Coding and Decoding Procedures}

To solve a problem by GA, the first task is to represent a solution of the problem as a chromosome, $P$. This representation is called coding. For combinatorial optimization, there are several representation methods, among which permutation-based representation (Gen and Cheng, 1997) and random-key (Bean, 1994) are often used. The procedure to change a chromosome $P$ into a phenotype solution with an objective value $f(P)$ is called decoding.

\subsection{Coding of an Arbitrary Solution}

A mixed chromosome, which includes both the carton packing order and the carton orientations, is adopted to represent a packing solution in this research. The mixed chromosome $P=\left(P_{1}, P_{2}\right)$ consists of two parts: $P_{1}=\left(\pi_{1}, \pi_{2}, \pi_{3}, \ldots, \pi_{N}\right)$ is a carton sequence, $\pi_{i} \in\{1,2,3, \ldots, N\}, i=1,2,3, \ldots, N ; P_{2}=\left(\gamma_{1}, \gamma_{2}, \gamma_{3}, \ldots, \gamma_{N}\right)$ is an orientation sequence, $\gamma_{i} \in\{1,2,3, \ldots, 6\}, i=1,2,3, \ldots, N$. Fig. 4 shows a sample chromosome for a problem with 10 cartons, where each carton $\pi_{i}$ in $P_{1}$ is given a corresponding orientation $\gamma_{i}$ in $P_{2}$.

Carton sequence $P_{1}$
\begin{tabular}{|llllllllll|llllllllll|} 
Orientation sequence $P_{2}$ \\
\hline 1 & 10 & 6 & 8 & 3 & 5 & 2 & 9 & 7 & 4 & 1 & 1 & 4 & 4 & 5 & 3 & 6 & 4 & 5 & 1 \\
\hline
\end{tabular}

Fig. 4 A sample mixed chromosome

\subsection{Decoding Procedure}

The decoding routine converts a chromosome into a feasible packing solution. Cartons in the given chromosome are processed sequentially, and their coordinates and relative positions are determined at the same time.

\subsubsection{Carton List and Reference Point List}

To decode a chromosome, two data lists, a carton list (c-list) and a reference point list (ref-point-list), are used to store the relevant data. A c-list corresponds to a chromosome. Each element $i$ in the c-list contains the following information: carton no. $\pi_{i}$, orientation no. $\gamma_{i}$, original size $\left(l_{i} \times w_{i} \times h_{i}\right)$, orientational dimensions $\left(l n_{i} \times w n_{i} \times h n_{i}\right)$ (derived from orientation no. $\gamma_{i}$ and original size), coordinates $\left(x_{i}, y_{i}, z_{i}\right)$ (to be determined), and relative positions (left-carton-no, front-carton-no, bottom-carton-no) (to be determined). Table 2 shows a sample c-list for the sample chromosome in Fig. 4. The coordinates and relative positions of the cartons are variables to be determined so that all cartons do not intersect with each other while maintaining a packing solution as compact as possible. When the coordinates and relative positions of the cartons in the c-list are determined through the decoding procedure (i.e. the packing procedure), the c-list is changed into a packing solution. 
Table 2. The sample carton list for the sample chromosome in Fig. 4

\begin{tabular}{|c|c|c|c|c|c|c|c|c|c|c|c|c|c|c|c|}
\hline & & & \multicolumn{3}{|c|}{ Original size } & \multicolumn{3}{c|}{ New dimensions } & \multicolumn{3}{c|}{ Coordinates } & \multicolumn{3}{c|}{ Relative positions } \\
\hline $\boldsymbol{i}$ & $\boldsymbol{\pi}_{\boldsymbol{i}}$ & $\boldsymbol{\gamma}_{\boldsymbol{i}}$ & $\boldsymbol{l}_{\boldsymbol{i}}$ & $\boldsymbol{w}_{\boldsymbol{i}}$ & $\boldsymbol{h}_{\boldsymbol{i}}$ & $\boldsymbol{I n}_{\boldsymbol{i}}$ & $\boldsymbol{w n}_{\boldsymbol{i}}$ & $\boldsymbol{h n}_{\boldsymbol{i}}$ & $\boldsymbol{x}_{\boldsymbol{i}}$ & $\boldsymbol{y}_{\boldsymbol{i}}$ & $\boldsymbol{z}_{\boldsymbol{i}}$ & left & front & bottom \\
\hline 1 & $\mathbf{1}$ & $\mathbf{1}$ & 36 & 36 & 36 & $\mathbf{3 6}$ & $\mathbf{3 6}$ & $\mathbf{3 6}$ & & & & & & \\
\hline 2 & $\mathbf{1 0}$ & $\mathbf{1}$ & 33 & 21 & 4 & $\mathbf{3 3}$ & $\mathbf{2 1}$ & $\mathbf{4}$ & & & & & & \\
\hline 3 & $\mathbf{6}$ & $\mathbf{4}$ & 40 & 31 & 21 & $\mathbf{3 1}$ & $\mathbf{2 1}$ & $\mathbf{4 0}$ & & & & & & \\
\hline 4 & $\mathbf{8}$ & $\mathbf{4}$ & 31 & 17 & 16 & $\mathbf{1 7}$ & $\mathbf{1 6}$ & $\mathbf{3 1}$ & & & & & & \\
\hline 5 & $\mathbf{3}$ & $\mathbf{5}$ & 54 & 40 & 21 & $\mathbf{2 1}$ & $\mathbf{5 4}$ & $\mathbf{4 0}$ & & & & & & \\
\hline 6 & $\mathbf{5}$ & $\mathbf{3}$ & 52 & 33 & 20 & $\mathbf{3 3}$ & $\mathbf{5 2}$ & $\mathbf{2 0}$ & & & & & & \\
\hline 7 & $\mathbf{2}$ & $\mathbf{6}$ & 59 & 39 & 20 & $\mathbf{2 0}$ & $\mathbf{3 9}$ & $\mathbf{5 9}$ & & & & & & \\
\hline 8 & $\mathbf{9}$ & $\mathbf{4}$ & 26 & 23 & 14 & $\mathbf{2 3}$ & $\mathbf{1 4}$ & $\mathbf{2 6}$ & & & & & & \\
\hline 9 & $\mathbf{7}$ & $\mathbf{5}$ & 31 & 31 & 17 & $\mathbf{1 7}$ & $\mathbf{3 1}$ & $\mathbf{3 1}$ & & & & & & \\
\hline 10 & $\mathbf{4}$ & $\mathbf{1}$ & 58 & 37 & 21 & $\mathbf{5 8}$ & $\mathbf{3 7}$ & $\mathbf{2 1}$ & & & & & & \\
\hline
\end{tabular}

Based on the concept of reference points in $\S 2.2$, a ref-point-list is adopted to store the information of the ref-points generated in the process of packing: coordinates, the type of the point, the temporary indices, occupied status. As stated earlier, an empty bin has one ref-point $(0,0,0)$, and the first carton is assigned to this point hence marked as "occupied". And then every time when carton $\pi_{i}$ with $l n_{i} \times w n_{i} \times h n_{i}$ is assigned at $\left(x_{i}, y_{i}, z_{i}\right)$, three new reference points, $\left(x_{i}+\ln _{i}, y_{i}, z_{i}\right),\left(x_{i}, y_{i}+w n_{i}, z_{i}\right)$ and $\left(x_{i}, y_{i}, z_{i}+h n_{i}\right)$, are generated and added to the ref-point-list as "not occupied yet". A feasible ref-point for the current carton to be packed is the one which ensures that the carton will not intersect with any cartons packed so far or the bin itself. If a ref-point is selected to assign a carton, this point may not be the final position of the carton because of the later parallel moves (introduced in $\$ 3.2 .2$ ), which try to move the carton along the $\mathrm{x}-, \mathrm{y}$ - and $\mathrm{z}$-axis to make the packing more compact.

To efficiently conduct the parallel moving, we classify the ref-points into three types: type $1,\left(x_{i}+\ln _{i}, y_{i}\right.$, $\left.z_{i}\right)$; type $2,\left(x_{i}, y_{i}+w n_{i}, z_{i}\right)$; and type $3,\left(x_{i}, y_{i}, z_{i}+h n_{i}\right)$. Furthermore, the just packed carton $\pi_{i}$ is recorded as the adjacent carton of these three points, $\left(x_{i}+\ln _{i}, y_{i}, z_{i}\right),\left(x_{i}, y_{i}+w n_{i}, z_{i}\right)$ and $\left(x_{i}, y_{i}, z_{i}+h n_{i}\right)$, which is convenient to determine the relative positions of the packed cartons. Therefore, each ref-point $k$ in the ref-point list should include the following information: coordinates $\left(x_{k}, y_{k}, z_{k}\right)$, the adjacent carton to this point (adj_carton), type no. (type 1, 2 or 3 ), two indices index 1 and index 2 (for reference point selection) and the assignment flag (as_flag).

\subsubsection{Parallel Moves, Relative Positions and Reference Point Selection}

Every time a carton is assigned to a ref-point, parallel moves can be performed for two purposes: one is to make the packing more compact by moving the carton as close as possible to the left, front and bottom of the bin; the other is to determine the relative positions of the carton packed.

Parallel moving was also conducted in $\mathrm{Wu}$ et al. (2010) where a carton was tried to move along $\mathrm{x}-, \mathrm{y}-$ and $\mathrm{z}$-axis in turn $(\mathrm{x} \rightarrow \mathrm{y} \rightarrow \mathrm{z})$ after it was packed on a ref-point. However, fixed-sequence moves along $\mathrm{x}-\mathrm{y}$ and z-axis might not be the optimal configuration for all cartons. The example in Fig. 5 illustrates the shortcomings of the simple sequential moves $x \rightarrow y \rightarrow z$. In Fig. 5, four cartons have been packed in the bin, 
assume ref-points 1, 2 and 3 are selected to assign a carton and then conduct parallel moves. Fig. 5(a) and (c) shows the situation of the moves $\mathrm{x} \rightarrow \mathrm{y} \rightarrow \mathrm{z}$ to the cartons assigned at ref-points 1, 2 and 3; Fig. 5(b) presents the result of the moves $\mathrm{y} \rightarrow \mathrm{x} \rightarrow \mathrm{z}$ to the cartons assigned at ref-points 1 and 2; and Fig. 5(d) displays the case of the moves $\mathrm{x} \rightarrow \mathrm{z} \rightarrow \mathrm{y}$ to the carton assigned at point 3 .

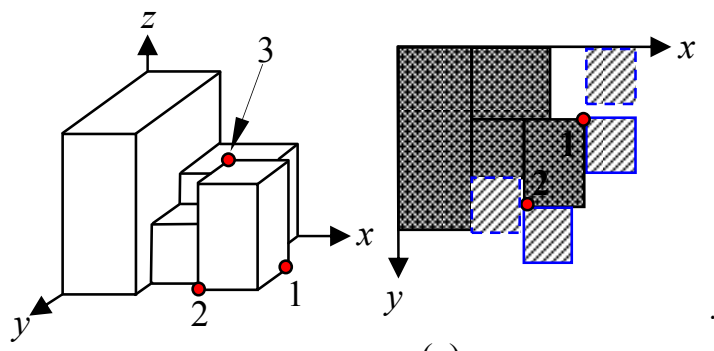

(a) $\mathrm{x} \rightarrow \mathrm{y} \rightarrow \mathrm{z}$

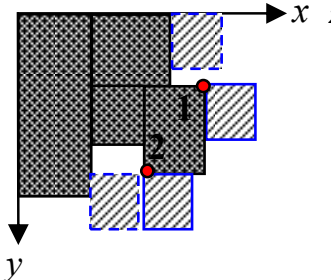

(b) $\mathrm{y} \rightarrow \mathrm{x} \rightarrow \mathrm{z}$

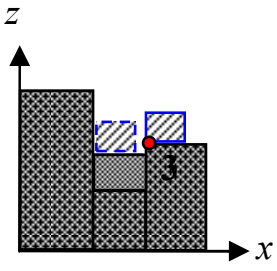

(c) $\mathrm{x} \rightarrow \mathrm{y} \rightarrow \mathrm{z}$

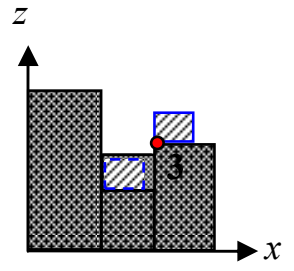

(d) $x \rightarrow z \rightarrow y$

Fig. 5 Different moves

From these cases we note that: (1) different sequence of parallel moves leads to different outcome; (2) one or two moves among the three moves in turn make no difference; (3) a further move may fill an additional gap; (4) one move may fail to fill any gap, but it can surely tell which carton is touched during the process.

Table 3. Different moves according to ref-point types

\begin{tabular}{|c|c|l|}
\hline Ref-point type & Moves in turn & \multicolumn{1}{|c|}{ Interpretation } \\
\hline Type 1 & $\mathrm{y} \rightarrow \mathrm{z} \rightarrow \mathrm{x}(\rightarrow \mathrm{z})$ & With a left carton, no need for $\mathrm{x}$ move at first. \\
\hline Type 2 & $\mathrm{x} \rightarrow \mathrm{z} \rightarrow \mathrm{y}(\rightarrow \mathrm{z})$ & With a front carton, no need for y move at first. \\
\hline Type 3 & $\mathrm{x} \rightarrow \mathrm{y} \rightarrow \mathrm{z}(\rightarrow \mathrm{x})$ & With a bottom carton, no need for $\mathrm{z}$ move at first. \\
\hline$\left(0,0, H_{c}\right)$ & $\mathrm{z}$ move only & On the top of the cage bin, no need for other moves. \\
\hline
\end{tabular}
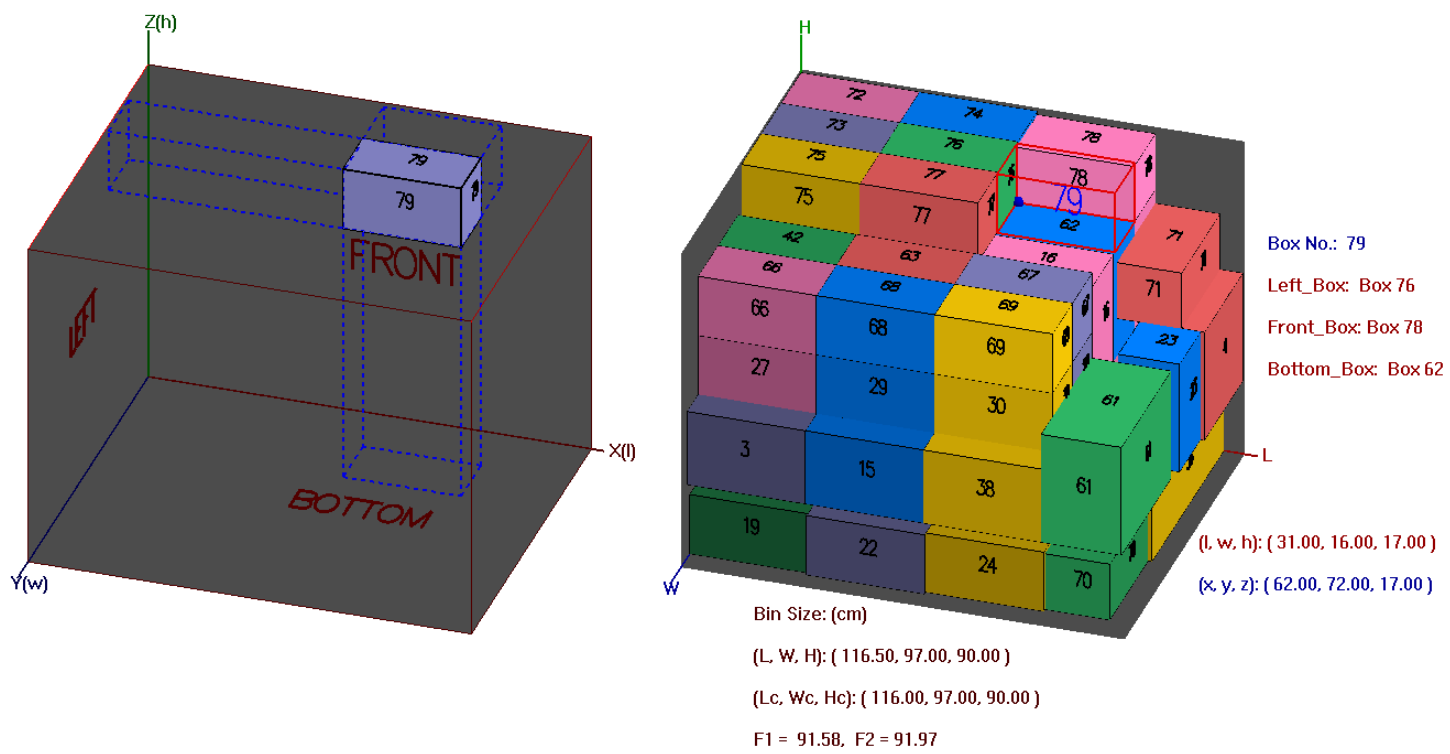

Fig. 6 Illustration of relative positions in packing

Based on the observation, a new parallel moving strategy is proposed as follows. First, we classify the 
ref-points generated by the packed cartons into three types as stated in \$3.2.1. We then conduct different moves to a carton in line with the type of the ref-point selected, as shown in Table 3.

Relative positions are intended to tell which cartons are touched on the left, in front and at the bottom of a carton (see Fig. 6), which are meaningful to guide the (especially manual) packing process. We denote the bin itself as carton " 0 " so that every carton packed in the bin is associated with three cartons to identify its relative positions: one on the left, one in front and one at the bottom.

For reference point selection, Wu et al. (2010) defined an index which we call index 1 in this research. For ref-point $k$, let $l_{c k}, w_{c k}, h_{c k}$ denote the length, width and height of the cage bin after the carton $\pi_{i}$ is placed at this point, $V_{i}$ denote the total volume of cartons packed so far including carton $\pi_{i}$, index 1 is defined as:

$$
\text { index } 1=\left(l_{c k} \times w_{c k} \times h_{c k}^{2}\right) / V_{i},
$$

which is a measure of the cage bin height over the fill rate of the current cage bin. In their work, the ref-point with the highest packing quality among the feasible ref-points is selected as the packing point for carton $\pi_{i}$. In Fig. 7, the third carton with $l n_{3} \times w n_{3} \times h n_{3}$ is being packed, and ref-points 3, 4, 5,6 and 7 are the feasible points, of which ref-points 3, 4 and 5 are obviously not so suitable as 6 and 7. Compared with ref-point 7, ref-point 6 has a higher value of $\boldsymbol{i n d e x} 1$ thus is selected to pack the third carton.
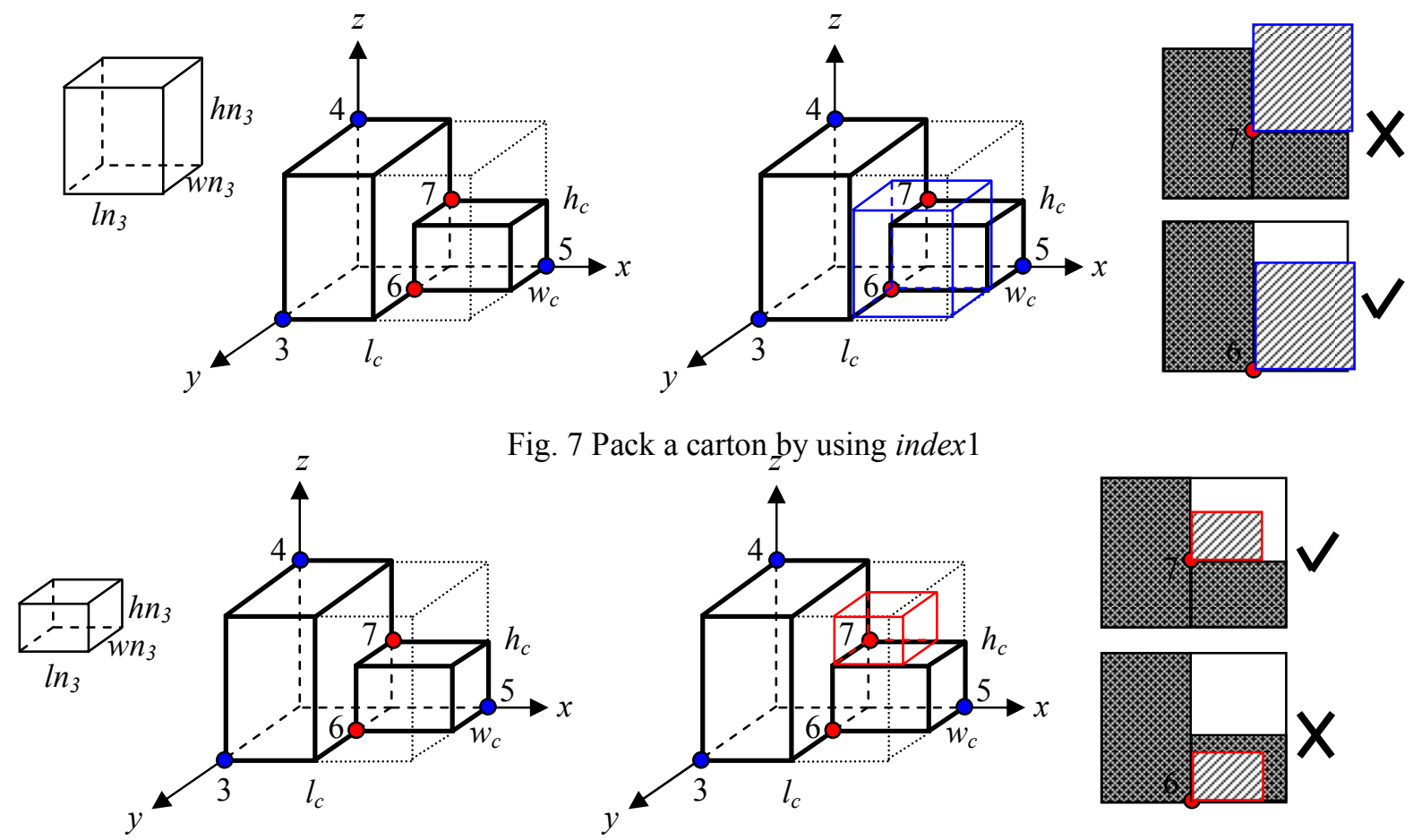

Fig. 7 Pack a carton by using index 1

Fig. 8 Pack a carton by using index 2

However, only using index 1 for ref-point selection is not comprehensive enough. In the case that there are several ref-points for packing carton $\pi_{i}$, whose cage bins are the same (i.e. when carton $\pi_{i}$ is assign to anyone of these points, it will not exceed the current cage bin), these points have the same value of index 1 . In this case, index 1 is not able to offer differentiating information for these points, and we need a new index to effectively select one of these points.

Assume that the coordinates of ref-point $k$ is $\left(x_{k}, y_{k}, z_{k}\right)$, carton $\pi_{i}$ has dimensions of $l n_{i} \times w n_{i} \times h n_{i}$, the 
current cage bin before packing carton $\pi_{i}$ has dimensions of $l_{c} \times w_{c} \times h_{c}$. Given that $\left(x_{k}+\ln _{i}\right) \leq l_{c},\left(y_{k}+w n_{i}\right) \leq w_{c}$, $\left(z_{k}+h n_{i}\right) \leq h_{c}$. A spare volume is defined as dynamic residual space with dimensions of $l_{s} \times w_{s} \times h_{s}$, where $l_{s}=$ $\left(l_{c}-x_{k}\right), w_{s}=\left(w_{c}-y_{k}\right)$ and $h_{s}=\left(h_{c}-z_{k}\right)$. The index 2 is defined as:

$$
\text { index } 2=\left(l_{s} \times w_{s} \times h_{s}^{2}\right) /\left(l n_{i} \times w n_{i} \times h n_{i}\right),
$$

which is a measure of the spare-height over the filled rate of the spare volume. In our work, the ref-point with the lowest index 2 among the feasible ref-points is selected as the packing point for carton $\pi_{i}$. Fig. 8 shows an example to select a ref-points using index2.

When packing a carton, we first choose a spare volume with lowest index2 to assign it; if no such a spare volume, then we select a ref-point with the highest packing quality index 1 ; if no ref-point is feasible to put the current carton, we then put it at $\left(0,0, H_{c}\right)$, where $H_{c}$ is the height of the current cage bin.

\subsubsection{Detailed Decoding Procedure}

Fig. 9 presents the detailed flowchart of the decoding procedure. To measure the quality of a packing solution, volume utilization (Bischoff and Ratcliff, 1995) is quite often used by the researchers, which is defines as:

$$
F_{1}=\left[V_{\text {total }} /(L \times W \times H)\right] \times 100 \%
$$

where $V_{\text {total }}$ is the total volume of the cartons, $(L \times W \times H)$ is the volume of the bin used. We also note that Loh and Nee (1992) used "packing density", which is defined as:

$$
F_{2}=\left[V_{\text {total }} /\left(L_{c} \times W_{c} \times H\right)\right] \times 100 \%
$$

where $\left(L_{c} \times W_{c} \times H\right)$ is the volume of the final cage bin, the smallest rectangular box enveloping all the cartons packed in the bin. 


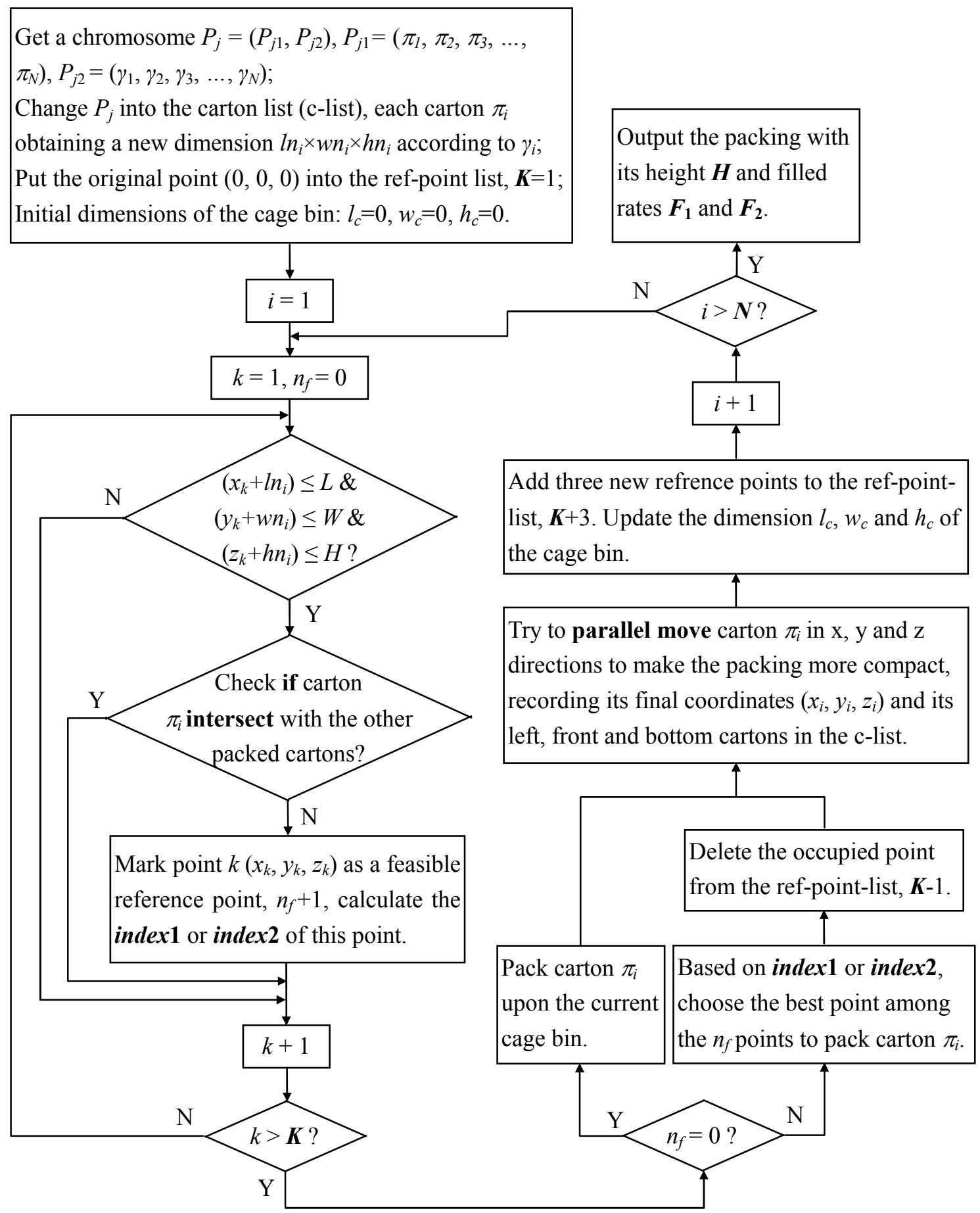

Fig. 9 The decoding procedure

In this work, both $F_{1}$ and $F_{2}$ are used to measure the quality of a packing. $F_{1}$ is equivalent to $H$, but $F_{1}$ is more distinctly to reveal the utilization rate of net space of the bin. Evidently, $F_{2}$ reflects the real tightness of the packing, hence should be used as one of the criteria to evaluate the performance the solution methods, regardless of the argument that $F_{2}$ overestimates the usual volume utilization (Bischoff and Ratcliff, 1995).

Table 4 presents the packing solution for the sample chromosome in Fig. 4, with the coordinates and relative positions available. 
Table 4. The packing solution for the sample chromosome in Fig. 4

\begin{tabular}{|c|c|c|c|c|c|c|c|c|c|c|c|c|c|c|}
\hline \multirow[b]{2}{*}{$i$} & \multirow[b]{2}{*}{$\pi_{i}$} & \multirow[b]{2}{*}{$\gamma_{i}$} & \multicolumn{3}{|c|}{ Original size } & \multicolumn{3}{|c|}{ New dimensions } & \multicolumn{3}{|c|}{ Coordinates } & \multicolumn{3}{|c|}{ Relative positions } \\
\hline & & & $l_{i}$ & $w_{i}$ & $h_{i}$ & $\ln _{i}$ & $w n_{i}$ & $h n_{i}$ & $x_{i}$ & $y_{i}$ & $z_{i}$ & left & front & bottom \\
\hline 1 & 1 & 1 & 36 & 36 & 36 & 36 & 36 & 36 & $\mathbf{0}$ & 0 & $\mathbf{0}$ & $\mathbf{0}$ & 0 & 0 \\
\hline 2 & 10 & 1 & 33 & 21 & 4 & 33 & 21 & 4 & $\mathbf{0}$ & 0 & 36 & $\mathbf{0}$ & $\mathbf{0}$ & 1 \\
\hline 3 & 6 & 4 & 40 & 31 & 21 & 31 & 21 & 40 & $\mathbf{0}$ & 36 & 0 & $\mathbf{0}$ & 1 & $\mathbf{0}$ \\
\hline 4 & 8 & 4 & 31 & 17 & 16 & 17 & 16 & 31 & 31 & 36 & $\mathbf{0}$ & 6 & 1 & $\mathbf{0}$ \\
\hline 5 & 3 & 5 & 54 & 40 & 21 & 21 & 54 & 40 & $\mathbf{0}$ & $\mathbf{0}$ & 40 & $\mathbf{0}$ & $\mathbf{0}$ & 10 \\
\hline 6 & 5 & 3 & 52 & 33 & 20 & 33 & 52 & 20 & 21 & 0 & 40 & 3 & 0 & 10 \\
\hline 7 & 2 & 6 & 59 & 39 & 20 & 20 & 39 & 59 & 54 & 0 & 0 & 5 & 0 & 0 \\
\hline 8 & 9 & 4 & 26 & 23 & 14 & 23 & 14 & 26 & 48 & 39 & $\mathbf{0}$ & 8 & 2 & 0 \\
\hline 9 & 7 & 5 & 31 & 31 & 17 & 17 & 31 & 31 & 36 & $\mathbf{0}$ & 0 & 1 & 0 & 0 \\
\hline 10 & 4 & 1 & 58 & 37 & 21 & 58 & 37 & 21 & 21 & 0 & 60 & 3 & $\mathbf{0}$ & 5 \\
\hline & & & & $L=$ & $W=$ & $H=$ & $F_{1}=$ & $3 \%$ & 7 & $V_{c}=$ & $7, F_{2}$ & 76.60 & & \\
\hline
\end{tabular}

\subsection{Sorting Heuristics and Random Search}

Sorting the cartons according to certain rules has demonstrated some advantages in providing better starting solutions for meta-heuristics. In fact, with the decoding procedure, we can conduct packing simulation experiments to test the sorting heuristics of ordering the cartons. For instance, we can compare the packing of the carton sequence in decreasing volume order and with various orientations. We also can randomly search a large number of random solutions (chromosomes) within very short computational time and select the best one for real packing. From the subsequent numerical experiments, we can see that the random search combined with a sorting heuristic demonstrates better performance in solving large-size packing problems than MILP model.

\section{The Improved GA (IGA)}

\subsection{Tailored Components of IGA}

\subsubsection{Generation}

At the beginning of the GA, an initial generation of chromosomes are often randomly generated. In IGA, a new mechanism for producing the initial generation is designed considering both "first fit decreasing" and diversity: (1) Six chromosomes with the same carton sequence in decreasing volume order, and each with an orientation sequence of the same orientation $\gamma_{j}, \gamma_{j}=1,2,3, \ldots, 6$. That means, for each chromosome, the cartons are packed in decreasing volume order and in the same orientation. Six orientations are exactly covered by the six chromosomes. (2) The remainder chromosomes are produced randomly as usual.

Let popsize denote the number of individuals in the initial generation, which is a critical parameter to control the solution quality, and is given according to problem size. At every iteration of GA, a new generation is re-produced through crossover, mutation and selection, and popsize is usually kept constant.

\subsubsection{Selection}

Selection is the process to select most of the better individuals in each generation for crossover, part of the generation (usually worse individuals) for mutation. Common selection methods include the 
roulette-wheel selection (Goldberg, 1989) and the tournament method (Goldberg and Deb, 1991). The tournament method is adopted in IGA due to the fact that in the tournament method the objective value of a chromosome can be directly used as the selection criterion and thus time-saving.

In selection, the crossover and mutation rates are two crucial performance parameters that influence the convergence speed of the GA. Assume the number of the chromosomes that are selected to crossover is xsize; the number of the chromosomes that are selected to mutate is msize. The ratio $C_{r}=x$ size/popsize is called crossover rate, often $C_{r} \in[0.5,0.9]$; and the ratio $M_{r}=$ msize/popsize called mutation rate, often $M_{r} \in[0.1$, 0.5]. In general, if $M_{r}$ increases, GA will converge slowly, thus has more chances to approach better solutions; but if $M_{r}$ is too large, GA tends to behave like a random search.

\subsubsection{Crossover}

Crossover is the process during which two parents generate two offspring, such that the children inherit a set of building blocks from each parent. Genetic operators are related to representation schemes. Poon and Carter (1995) presented a survey of crossover operators for ordering applications. Regarding the permutation-based representation, the following crossover operators have been widely used: partially matched crossover (PMX) intending to keep the absolute positions of elements and linear order crossover (LOX) intending to respect relative positions. In IGA, PMX is basically adopted for the carton sequences; but for the orientation sequences, concomitant PMX is performed, so that the orientation of a carton is kept unchanged, as shown in Fig. 10. The orientation change for a carton is implemented through the following mutation operator.

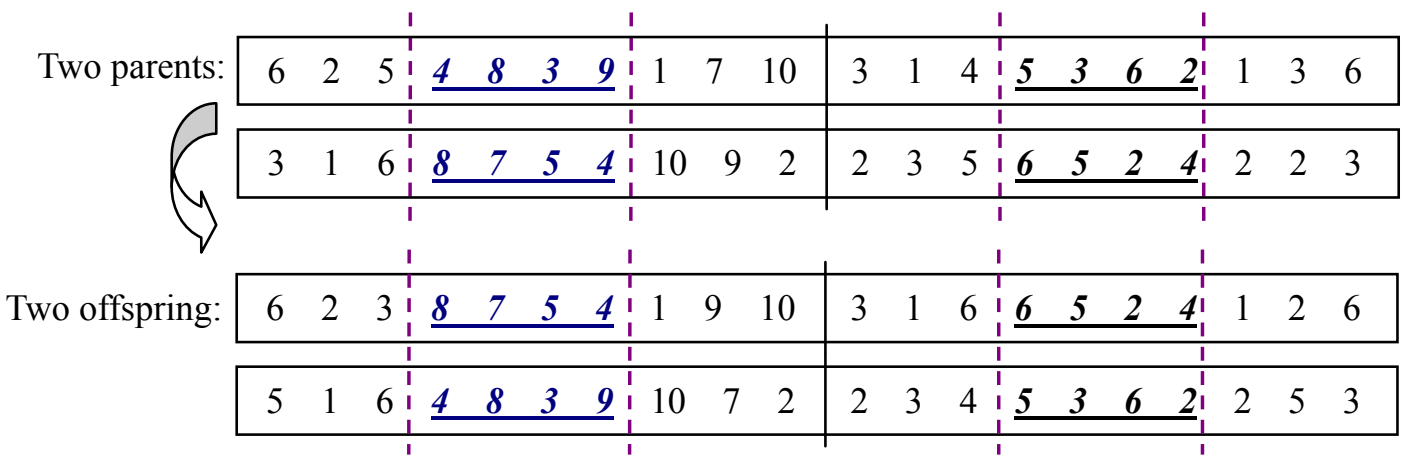

Fig. 10 Illustration of the process of concomitant PMX

\subsubsection{Mutation}

Mutation is the process to change some genes in a chromosome and produce a new chromosome. There are also a number of methods to mutate (Gen and Cheng, 1997), such as reversion, swap and insertion.

He (2007 and 2009) has conducted a comparative study on reversion and swap mutations in solving symmetrical and asymmetrical combinatorial problems, such as the traveling salesman problem (TSP) and the zero-wait scheduling problems (ZWSP). According to our experience, it is evident that the swap mutation is favorable to the problems with asymmetrical feature, especially in solving the large-size instances. The 3D-BPP is of highly asymmetrical feature, hence the swap mutation is found to be advantageous.

The mutation in this work has two functions: one is to change the order of the cartons to be packed; the 
other is to assign new orientations for a carton. Following reversion or swap, random change of orientations is conducted, making it possible for each carton to be packed in different orientations, and allowing the algorithm to select the right orientation for a carton. Fig. 11 illustrates the mutation procedures. Considering the following random change of orientations, the reversion or swap can be only carried out to the carton sequence.

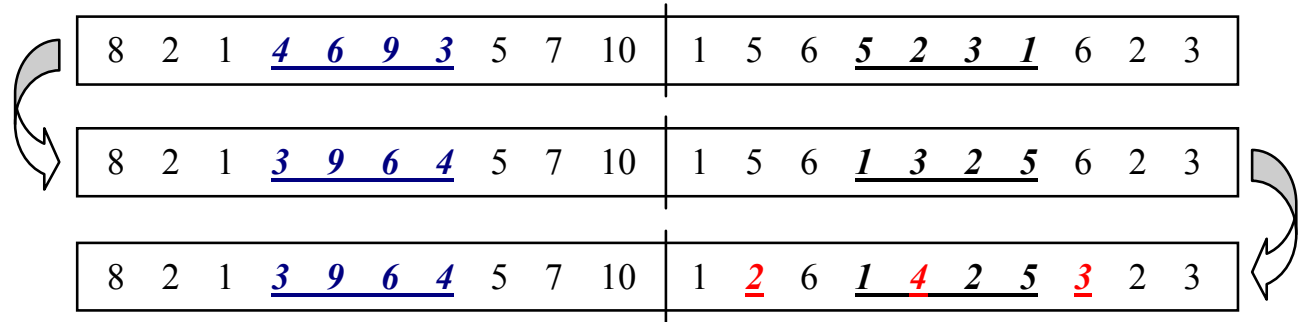

(a) Reversion and random change of orientations

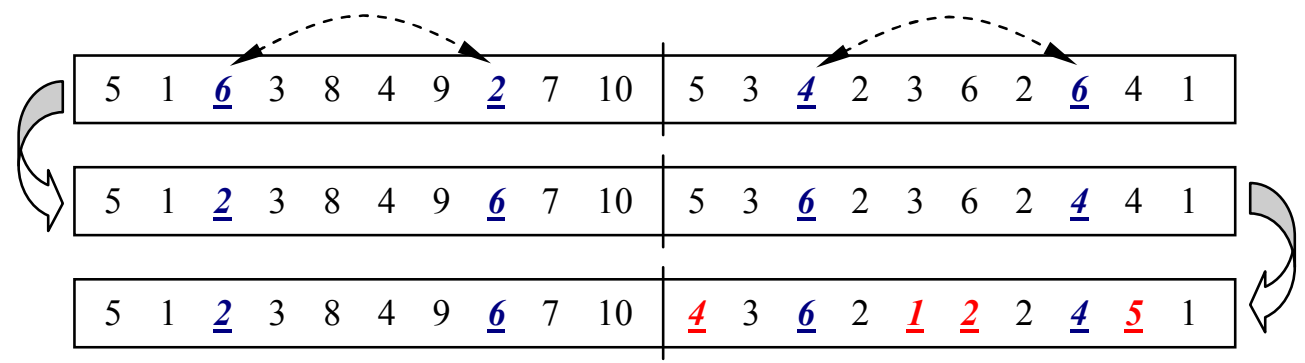

(b) Swap and random change of orientations

Fig. 11 Illustration of the process of mutation

\subsubsection{Termination Condition}

Termination condition for IGA is that the algorithm stops when the objective value difference between the worst chromosome and the best one in the current generation is equal to or less than a small value, like 0.1 or 0.01 , which is dependent on the precision of the objective values.

\subsection{Procedure of IGA}

With GA components customized, the GA procedure is laid out as follows:

Step 1. Initial generation and evaluation: Produce an initial generation comprising popsize chromosomes, and evaluate all the chromosomes using the decoding procedure.

Step 2. Selection: Select xsize better chromosomes in each generation to crossover, and msize worse chromosomes to mutate.

Step 3. Crossover and Mutation: Conduct concomitant PMX to the xsize better chromosomes generating xsize new chromosomes. Conduct mutation to the msize worse chromosomes generating msize new chromosomes.

Step 4. Evaluation and new generation: The offspring (xsize+msize new chromosomes) from crossover and mutation are decoded. Sort the parents and the offspring according to their objective values, and select the former popsize best chromosomes as the new generation.

Step 5. Test the termination condition: If the termination condition is met, then go to Step 6; else go 
to Step 2 for a new iteration.

Step 6. Output the results: Output the best chromosome and its corresponding packing solution.

\subsection{Solution of SM00 by IGA}

IGA, inclusive of the decoding procedure is implemented in $\mathrm{C}$ language. Table 5 presents the results of 10 runs of IGA for SM00, where the parameter settings are popsize $=200, x$ size $=160$, msize $=40$, with swap mutation used, and $t$ denote the iterations in each test $l$.

To evaluate the performance of meta-heuristics, a number of computational tests are carried out. Each test is one run of the algorithm with one final solution. The relative deviation (denoted by $R D_{l}$ ) from the best solution of the tests is used to evaluate each test, which is defined as:

Table 5. The results of 10 tests of IGA for SM00

$$
R D_{l}=\left[\left(H_{l}-H_{\text {best }}\right) / H_{\text {best }}\right] \times 100 \% \text {, }
$$

where $H_{l}$ is the objective value of the solution in test $l, H_{\text {best }}$ is the best of the tests. A lower average relative deviation is generally expected.

\section{The Global Search Framework for IGA}

Besides the improvements on the genetic algorithm, the global search framework (GSF, He and Hui, 2010) is adopted to achieve better solutions for the large-size and highly heterogeneous packing problems. In this framework, both evolved solutions and dynamic statistic information (the so-called evolutionary gradient) are harnessed to guide the global search.

As well known, single meta-heuristic is problem dependent, its robustness is limited, and premature convergence is another main drawback, especially for cases of large-scale problems. Hybridization, parallelization and other search frameworks are the main effective measures to enhance the robustness and to overcome the drawback of premature convergence. The proposed framework aims to address two issues: the first goal is to solve larger problems within reasonable computational time; the second is to make the algorithm more robust and less calibration effort. In fact, this framework has realized some parallel ideas in the sequential environment.

It is well known that every run of the exact model for the same problem obtains the same solution; but different runs of a meta-heuristic algorithm may obtain different solutions. That is why we often run the meta-heuristic algorithm several times for the same problem and select the best solution for use. However, in practical application, the user is more willing to run the packing program for only one time and then obtain the expected packing solution. 
The distinction between the solutions from a number of computational tests is the impetus for us to pursue much better solutions. In other words, the existing distinction implies that much better solutions are possible if more computational tests are conducted. At the moment of seeing the distinction between the solutions, what we should consider is how to effectively conduct the subsequent computational tests and when to stop computing. Regarding these two questions, we proposed the concept of evolutionary gradient and then devised a global search framework (He and Hui, 2010). In this framework, when the evolutionary gradient vanishes, the algorithm stops. In this work, five scenarios of GSF are devised and tested, and the best scenario is selected for the practical 3D packing problem.

\subsection{Evolutionary Gradient}

As well known, the concept of gradient is adopted in numerical methods, where the gradient is regarded as the criterion to determine the termination of the algorithm - if the gradient turns to be zero, the optimum or local optimum is deemed to have been found. Similarly, in evolutionary algorithms, the distinction between the solutions obtained so far from computational tests can be treated as the criterion to determine the termination of the algorithms, thus the concept of evolutionary gradient is proposed.

When a number of computational tests are carried out to evaluate the given algorithm, the relative deviation $(R D)$ of each test can be calculated with respect to the current best solution:

$$
R D(\%)=[(\text { objective value }- \text { current best }) / \text { current best }] \times 100 \% \text {. }
$$

Let $N_{L}$ denote the number of computational tests of the single IGA. The mean relative deviation is defined as evolutionary gradient, denoted by $E_{d e v}$ :

$$
E_{d e v}=\frac{1}{N_{L}} \sum_{l=1}^{N_{L}} R D_{l}
$$

where $R D_{l}$ is the relative deviation of test $l$ calculated by Eq. 7. In fact, $E_{d e v}$ can be regarded as an important sign which indicates whether it is necessary to conduct more computational tests for better solutions. If the $N_{L}$ tests obtain solutions with the same objective value, then $E_{d e v}=0$, which implies that it is not necessary to carry out more tests; otherwise, $E_{d e v}>0$, which indicates the probability of better solutions through additional computational tests. The initial $N_{L}$ depends on the problem size. For large-size problems, we usually witness $E_{d e v}>0$, and additional runs are expected to provide better solutions. The additional $N_{L}$ can be determined by the subsequent Eq. 9 .

\subsection{The Global Search Framework}

Based on the concept of evolutionary gradient, three phases of computational tests are organized. In phase 1, which is called rough search, $N_{L}$ tests of IGA are carried out to see whether the evolutionary gradient exists $\left(E_{d e v}>0\right)$. If yes, go on with phase 2 which consists of one or more refining searches, each refining search involves $N_{L}=f\left(E_{d e v}\right)$ tests of IGA, until the evolutionary gradient turns to be zero $\left(E_{d e v}=0\right)$. Despite the zero evolutionary gradient approached at the end of phase 2, phase 3 can follow with further ambition to find more refined solutions. In phase 3, if no new solution is found, only one refining search is to 
be executed; otherwise, with new solution(s) obtained, several refining searches are to be conducted until the new evolutionary gradient becomes zero. The rough search and refining searches can be considered as local searches, while the whole organization of the three phases is called the global search framework. Each rough search or refining search is seen as a global iteration, denoted by $g$; and a single IGA test as a local iteration, denoted by $l$.

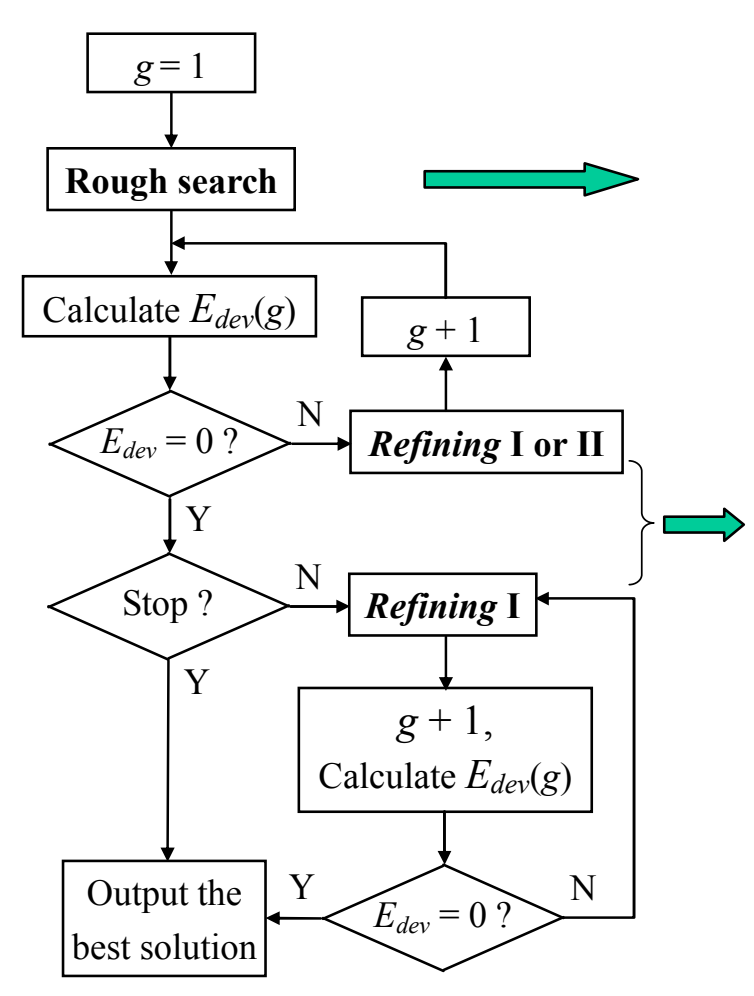

(a) Global search framework

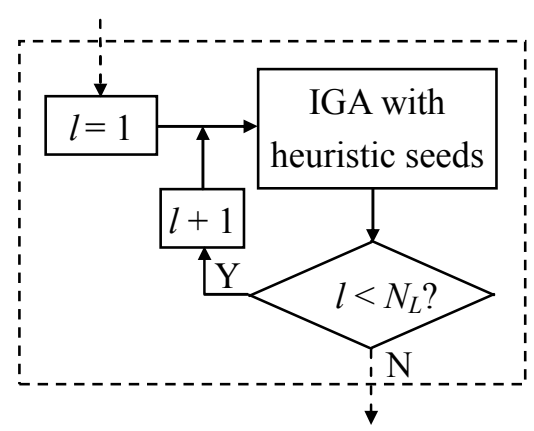

(b) Rough search

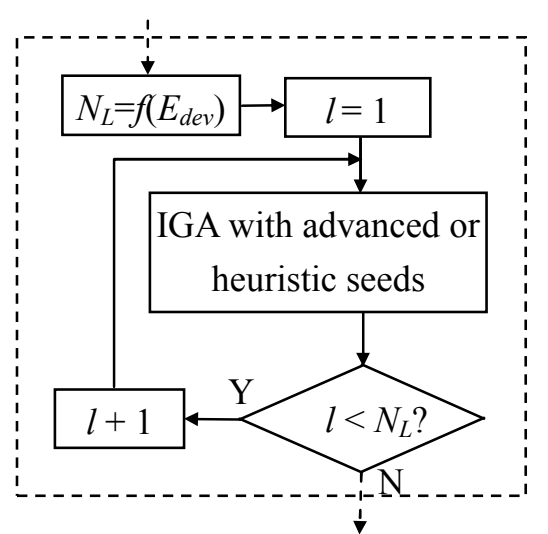

(c) Refining search

Fig. 12 Flow chart of the global search framework

The procedure of the GSF is shown in Fig. 12(a). The rough search, as shown in Fig. 12(b), comprises $N_{L}$ tests of IGA, each of which starts with an initial generation including the six heuristic chromosomes and (popsize-6) random chromosomes, where the six heuristic chromosomes can be treated as excellent "breed" or "seeds" to expedite the evolution. Each refining search, as shown in Fig. 12(c), comprises $N_{L}=f\left(E_{d e v}\right)$ tests of IGA, each test starts with an initial generation including advanced chromosomes from last global iteration and random chromosomes. $N_{L}=f\left(E_{d e v}\right)$ can be determined by Eq. 9 ,

$$
f\left(E_{d e v}\right)= \begin{cases}N_{L L}\left(1+\frac{1}{E_{d e v}}\right), & \text { if } \quad E_{d e v} \geq \frac{N_{L L}}{N_{L U}-N_{L L}} ; \\ N_{L U}, & \text { else }\end{cases}
$$

where $N_{L L}$ and $N_{L U}$ are respectively the lower and upper bounds of the number of IGA tests. $N_{L}$ increases with the decreasing of $E_{d e v}$, but has an upper bound. $N_{L}$ may also be set as a constant parameter in the search process. In this work, $N_{L}$ is set as a constant number.

It should be emphasized that, in addition to GAs, other hybrid solution methods can also be incorporated into the GSF. Using such a framework, much better solutions can be obtained in succession, 
until the evolutionary gradient vanishes.

\subsection{Scenarios of GSF Considering Evolution Speed}

The "refining $I$ " and "refining II" in Fig. 12 are two kinds of refining searches with different breed of selection strategies. For refining $I$, only the best solution found in iteration $g$ is put into the initial generation of each test in iteration $g+1$; but for refining $I I$, all the $N_{L}$ evolved solutions obtained in iteration $g$ are put into the initial generation of each test in iteration $g+1$. For refining $I I$, considering $N_{L}$ advanced seeds introduced into a new evolutionary environment, it seems that the six heuristic seeds are not necessary to the coming global iterations, and that $N_{L}$ can be reduced to a relative smaller number, both of which can expedite evolution or solution speed. With these considerations, the scenarios presented in Table 6 are laid out for simulation experiments to determine which one is the best. Subsequent numerical experiments demonstrate that, in refining $I I$, indeed $N_{L}$ can be reduced to a relative smaller number, but the six heuristic seeds are still vital to the coming global iterations.

Table 6. Scenarios of GSF for simulation experiments

\begin{tabular}{|c|c|c|c|c|}
\hline Scenario & Option of phases 1,2 or 3 , refining I or II & $N_{L}$ & Heuristic seeds or not & Performance \\
\hline GGA1 & Phase $1+2$ (refining I): slow and nice solution & 30 & Yes & $* * *$ \\
\hline GGA2 & Phase $1+2$ (refining $I I)$ : fast & 10 & No & $* *$ \\
\hline GGA3 & Phase $1+2$ (refining $I I)$ : fast & 20 & No & $* * *$ \\
\hline GGA4 & Phase $1+2$ (refining $I I)$ : fast and nice solution & 20 & Yes & $* * * *$ \\
\hline GGA5 & Phase $1+2$ (refining $I I)+3$ (refining $I)$ & 20 & Yes & $* * * * *$ \\
\hline
\end{tabular}

Furthermore, one may doubt the necessary existence of phase 3 in GGA5. For easy instances phases 1 and 2 are sufficient to obtain satisfactory solutions, indeed phase 3 additionally leads to another refining search wasting some CPU time. However, for hard instances phase 3 gives rise to more chances to find much better solutions. As known from the computational experiences in exact methods, even if the MILP model has found the best solution to an instance, nevertheless, a long time is still needed to prove the optimality. Compared with such a long time, the time for an additional refining search in phase 3 is very short.

\subsection{Solutions of SM00 by GSF under Diverse Scenarios}

Table 7 presents solutions of SM00 obtained under the various scenarios. GGA1 finds the best over a longer time because of the bigger $N_{L}$ and only one advanced seed for further evolution. GGA2 has a short time due to the smaller $N_{L}$ and $N_{L}$ advanced seeds for further evolution, but with a dissatisfactory solution. The difference of GGA3 from GGA2 is only that $N_{L}$ is doubled, thus the solution is improved. In GGA2 and GGA3, with $N_{L}$ advanced seeds for the coming global iterations, the evolution speed has indeed been expedited. The difference of GGA4 from GGA3 is that, six heuristic seeds together with $N_{L}$ advanced seeds are used for the coming global iterations, consequently further enhancing the evolution speed. GGA5 with the merits of both GGA4 (fast speed) and GGA1 (ability for better solution) has found the best solution in shorter time. 
Table 7. Solutions of SM00 by GSF under diverse scenarios

\begin{tabular}{|c|c|c|c|c|}
\hline Scenario & $\boldsymbol{N}_{\boldsymbol{L}}$ & $\boldsymbol{G}$ & CPU time $\boldsymbol{C T}$ (s) & $\boldsymbol{H}$ \\
\hline GGA1 & 30 & $1+2=3$ & 9.70 & 70 \\
\hline GGA2 & 10 & $1+2=3$ & 2.91 & 72 \\
\hline GGA3 & 20 & $1+2=3$ & 5.31 & 71 \\
\hline GGA4 & 20 & $1+2=3$ & 4.95 & 71 \\
\hline GGA5 & 20 & $1+2+1=4$ & 7.20 & 70 \\
\hline
\end{tabular}

\section{Computational Experiments}

In this section, we apply the proposed methods to the cases solved in Wu et al. (2010), and expand the experiments to some larger and more heterogeneous new cases. Moreover, GGA5 is tested with the benchmark instances BR01 10 (Bischoff and Ratcliff, 1995).

\subsection{Problem Data}

In $\mathrm{Wu}$ et al. (2010), 10 packing cases of small bins $\left(L \times W \times H_{\max }=80 \times 58 \times 95\right.$ for SM04 and SM05, and $L \times W \times H_{\max }=78 \times 57 \times 95$ for other small cases $)$ and 27 cases of big bins $\left(L \times W \times H_{\max }=116.5 \times 97 \times 95\right)$ were collected from a company where the workers manually pack cartons in the bin based on a rule of thumb and their own packing experience. In these cases, there exist 13 types of cartons, each case involves no more than 9 types of cartons, and the number of cartons to be packed into a bin is no more than $40(N \leq 40)$. We denote the original GA in $\mathrm{Wu}$ et al. (2010) as OGA.

The new cases in this work include 6 packing cases of small bins $\left(L \times W \times H_{\max }=80 \times 58 \times 95\right)$ and 12 cases of big bins $\left(L \times W \times H_{\max }=116.5 \times 97 \times 95\right)$. In contrast to the old small-bin cases which contain $3 \sim 5$ carton types, the new small-bin packing involves 8 11 carton types; and each new big-bin case involves 7 13 carton types instead of 2 9 carton types for the old big-bin cases. For the new cases, up to 40 cartons are loaded into a small bin, and up to 80 cartons are packed into a big bin.

To evaluate the performance of the methods for more heterogeneous instances, two particular big-bin packing cases, BM40 and BM41, are tested. In BM40 and BM41, 20 cartons to be packed involve 20 carton types, i.e., each carton belongs to a different carton type.

\subsection{Algorithm Parameter Setting}

The IGA and the global search framework are coded in C language and compiled with Microsoft Visual $\mathrm{VC}++$ 8.0. All the numerical experiments are conducted on a computer with an Intel $\mathrm{T} 75002.20 \mathrm{GHz}$ Duo CPU and 1.96GB RAM.

Parameter setting is an important issue which impacts the performance. In the GA, the setting of the population size (denoted by popsize) in a generation depends on the problem size. The crossover rate $\left(C_{r}\right)$ and mutation rate $\left(M_{r}\right)$ are two crucial factors that influence solution quality and convergence speed. In the GSF, the number $\left(N_{L}\right)$ of IGA tests within a global iteration is also an important parameter to determine the solution time and quality.

In OGA, $M_{r}$ is given differently according to the mutation methods, reversion (Rvs) or swap (Swp), and 
a fixed number of iterations (denoted by iter.) is set for each OGA test. Specifically, in OGA with Rvs mutation, (popsize, $C_{r}, M_{r}$, iter. $)=(120,0.9,0.5,200)$ for small-bin cases $(\mathrm{SM})$, and $(200,0.9,0.5,500)$ for big-bin cases (BM); in OGA with swap mutation, (popsize, $C_{r}, M_{r}$, iter.) $=(120,0.9,0.9,200)$ for SM, and $(200,0.9,0.9,500)$ for BM. A notable setting of $M_{r}$ is that it was set up to 0.9 ! The best solution by OGA is selected from 20 computational tests.

In IGA, $M_{r}$ has been tuned from 0.1 to 0.5 , with decreasing convergence speed, but not improving the solution quality so much, thus $M_{r}$ is given as 0.2 . Due to the termination condition of until-no-improvement adopted in IGA, the computation of the algorithm stops at the iteration of "no improvement". So in IGA, (popsize, $\left.C_{r}, M_{r}\right)=(200,0.8,0.2)$ for $\mathrm{SM}$, and $(400,0.8,0.2)$ for BM. The best solution by IGA is also selected from 20 computational tests.

In the different scenarios of GSF, (popsize, $\left.C_{r}, M_{r}\right)=(200,0.7,0.3)$ for SM, and $(400,0.7,0.3)$ for BM, with a little change of $C_{r}$ and $M_{r}$ to allow the algorithms to search more combinations of carton orientations. $N_{L}$ is set as 30 for GGA1, 10 for GGA2, 20 for GGA3, GGA4 and GGA5.

We also list the search results by the MILP, the manual packing (when possible) and a controlled random search. The MILP model is limited by a running time of 7200 seconds (two hours). In the random search (Rdm) combined with sorting heuristics based on the decoding procedure, 500 feasible solutions are searched for SM, 1000 for BM, including the six heuristic solutions.

\subsection{Results and Analysis}

With the parameter setting, numerical experiments have been performed. The comparative study is carried out from three aspects: the packing height $(H)$, filled rates $\left(F_{1}\right.$ and $\left.F_{2}\right)$ and computational time $(C T)$. Tables 8 and 9 show the best solutions $(H)$ of the cases obtained via a range of methods or scenarios. The best solution for an instance by OGA or IGA is selected from 20 computational tests, so the computational time for OGA or IGA is the total running time of the 20 tests. The other methods, including the random search, MILP and the scenarios of GSF, obtain the best or optimal solution for an instance at the end of their execution. Tables A-1 to A-5 in Appendix A provide the corresponding filling rates and computational times of these methods.

\subsubsection{Comparison of Packing Height}

From Tables 8 and 9, the following observations can be noted:

(1) Random search vs. manual packing. For the old cases, the manual packing heights are presented in Table 8. Compared with the manual packing, the random search has found better solutions for 22 cases among the 37 cases. Moreover, from filled-rate comparison (see Table A-1), it is noted that the random search gets a higher average $F_{1}$ than the manual packing. The random search is completed within one second for the old cases, or within 3 seconds for new cases (see Tables A-4 and A-5). For the moderate size instances (BM13 27), the random search approaches almost the same average $F_{1}$ as the manual packing. Hence, for the new cases where the manual packing data are not available, the results by the random search are treated as the ones by the manual packing. 
(2) Reversion mutation vs. swap mutation: Both in OGA and IGA, the two mutation methods have compared, and corresponding results are presented in Tables 8 and 9 from which it is verified again that swap mutation is more favorable to the solution quality of the algorithms.

(3) IGA(swp) vs. OGA(swp). IGA(swp) performs better than OGA(swp) both in solution quality and speed. For 31 cases among the 55 cases, IGA(swp) finds better solutions than OGA(swp). Only for BM24, IGA(swp) obtains a worse solution than OGA(swp).

(4) Different scenarios of GSF: Five scenarios of GSF as shown in Table 6 are also tested with all the cases to reveal their performance in solving diverse instances. The experiment results show that GGA1 and GGA4 have complementary merits: GGA1, further refining searches (local search actually) with one evolved solution consecutively, can find good solutions but with longer search time; GGA4, further refining searches with a number of evolved solutions consecutively, can obtain good solutions with higher speed. GGA5, first conducting refining searches with a number of evolved solutions and then with one evolved solution, has the merits of both GGA4 and GGA1, thus has found new solutions for most of the cases. For SM04, SM06 and SM10, GGA5 finds the same proven optimal solutions as MILP model, but the other GA-based methods have not found these solutions.

(7) GGA5 vs. GAs. For most of the instances, the GGA5 has achieved better solutions than OGA (see Tables 8 and 9, better solutions by GGA5 vs. OGA are marked with ${ }^{\#}$ ), but the computational times are much shorter (see Tables A-4 and A-5). Only the solution $(H=97)$ for SM15 by GGA5 exceeds $H_{\max }$, leaving this instance as a challenge for coming work. Furthermore, compared to IGA, GGA5 has achieved better solutions for almost all of the new cases (see Table 8, better solutions by GGA5 vs. IGA are marked with ${ }^{\text {s), }}$ which demonstrates that the GSF actually helps improve the solution quality, especially in solving the large-size cases. 
Table 8. The best solutions (heights) of the old cases by different methods MILP

\begin{tabular}{|c|c|c|c|c|c|c|c|c|c|c|c|c|c|}
\hline & \multirow[b]{2}{*}{$N$} & \multirow[b]{2}{*}{ Manual } & \multirow[b]{2}{*}{ Rdm } & \multirow[b]{2}{*}{ MILP $^{\&}$} & \multicolumn{2}{|c|}{ OGA } & \multicolumn{2}{|c|}{ IGA } & \multicolumn{5}{|c|}{ GSF } \\
\hline & & & & & Rvs & Swp & Rvs & Swp & GGA1 & GGA2 & GGA3 & GGA4 & GGA5 \\
\hline SM01 & 8 & 91 & 92 & $80 *$ & 80 & 80 & 80 & 80 & 80 & 80 & 80 & 80 & 80 \\
\hline SM02 & 8 & 56 & 40 & $37 *$ & 37 & 37 & 37 & 37 & 37 & 37 & 37 & 37 & 37 \\
\hline SM03 & 9 & 69 & 53 & $47 *$ & 47 & 47 & 47 & 47 & 47 & 47 & 47 & 47 & 47 \\
\hline SM04 & 9 & 91 & 103 & 82 & 83 & 83 & 83 & 83 & 82 & 83 & 83 & 83 & $82^{\#}$ \\
\hline SM05 & 10 & 56 & 61 & $52 *$ & 52 & 52 & 52 & 52 & 52 & 52 & 52 & 52 & 52 \\
\hline SM06 & 12 & 53 & 48 & $38^{*}$ & 40 & 40 & 40 & 40 & 40 & 40 & 40 & 40 & $38^{\#}$ \\
\hline SM07 & 17 & 70 & 62 & 62 & 62 & 62 & 62 & 62 & 62 & 62 & 62 & 62 & 62 \\
\hline SM08 & 17 & 91 & 77 & 71 & 68 & 69 & 64 & 62 & 62 & 64 & 62 & 62 & $62^{\#}$ \\
\hline SM09 & 19 & 71 & 62 & 62 & 52 & 52 & 51 & 51 & 51 & 51 & 51 & 51 & $50^{\#}$ \\
\hline SM10 & 21 & 85 & 78 & 62 & 64 & 63 & 64 & 63 & 62 & 63 & 62 & 62 & $62^{\#}$ \\
\hline BM01 & 5 & 91 & 84.1 & $84.1 *$ & 84.1 & 84.1 & 84.1 & 84.1 & 84.1 & 84.1 & 84.1 & 84.1 & 84.1 \\
\hline BM02 & 11 & 91 & 99 & 83.1 & 84.1 & 84.1 & 84.1 & 84.1 & 84.1 & 84.1 & 84.1 & 84.1 & 84.1 \\
\hline BM03 & 12 & 45 & 47 & $32 *$ & 32 & 32 & 32 & 32 & 32 & 32 & 32 & 32 & 32 \\
\hline BM04 & 13 & 91 & 98 & 77.4 & 83.4 & 83.4 & 83.4 & 77.4 & 77.4 & 79 & 77.4 & 77.4 & $77.4^{\#}$ \\
\hline BM05 & 15 & 91 & 90 & 79 & 80 & 79 & \begin{tabular}{|l|}
80 \\
\end{tabular} & 79 & 78 & 79 & 79 & 78 & $78^{\# \$}$ \\
\hline BM06 & 16 & 67 & 47 & 40 & 40 & 40 & 40 & 40 & 40 & 40 & 40 & 40 & 40 \\
\hline BM07 & 21 & 48 & 40 & 40 & 38 & 40 & 38 & 38 & 37 & 37 & 37 & 37 & $37^{\# \$}$ \\
\hline BM08 & 22 & 59 & 54 & 53 & 53 & 51 & 53 & 51 & 48 & 51 & 50 & 48 & $48^{\# \$}$ \\
\hline BM09 & 23 & 94 & 80 & 82 & 71 & 72 & 72 & 71 & 71 & 71 & 71 & 71 & $71^{\#}$ \\
\hline BM10 & 23 & 91 & 62 & 63.1 & 61 & 61 & 61 & 61 & 61 & 61 & 61 & 61 & 61 \\
\hline BM11 & 25 & 91 & 80.1 & 78 & 73.4 & 77.4 & 73.4 & 73.4 & 73.4 & 73.4 & 73.4 & 73.4 & $73.4^{\#}$ \\
\hline BM12 & 26 & 94 & 66 & 68 & 61 & 61 & 61 & 61 & 61 & 61 & 61 & 61 & 61 \\
\hline BM13 & 27 & 83 & 85 & 78 & 73 & 71 & 69 & 69 & 68 & 70 & 69 & 68 & $68^{\# \$}$ \\
\hline BM14 & 28 & 95 & 94 & 88.4 & 79 & 78.4 & 78.4 & 78.4 & 78.4 & 80 & 78.4 & 78.4 & 78.4 \\
\hline BM15 & 29 & 91 & 94 & 87 & 77 & 75 & 75 & 74 & 74 & 74 & 73 & 73 & $73^{\# \$}$ \\
\hline BM16 & 29 & 63 & 54 & 52 & 48 & 48 & 47 & 47 & 47 & 47 & 47 & 47 & $47^{\#}$ \\
\hline BM17 & 29 & 91 & 101 & 88.4 & 82 & 80 & 79 & 78.4 & 78.4 & 79 & 80 & 80 & $78.4^{\#}$ \\
\hline BM18 & 30 & 70 & 70 & 73.4 & 67 & 63.1 & 63 & 63 & 63 & 63 & 63 & 63 & $63^{\#}$ \\
\hline BM19 & 30 & 94 & 78.4 & 79 & 63.4 & 63.1 & 63.4 & 63.1 & 63 & 63 & 63 & 63 & $63^{\# \$}$ \\
\hline BM20 & 30 & 94 & 94 & 87 & 80 & 79 & 79 & 79 & 78 & 80 & 78 & 78 & $78^{\#}$ \\
\hline BM21 & 30 & 95 & 94 & 87 & 82 & 78.4 & \begin{tabular}{|l|}
78.4 \\
\end{tabular} & 78.4 & 78.4 & 78.4 & 78.4 & 78.4 & 78.4 \\
\hline BM22 & 31 & 91 & 85 & 87 & 78 & 78 & 78 & 78 & 77 & 78 & 78 & 77 & $77^{\# \$}$ \\
\hline BM23 & 33 & 95 & 94 & 93.4 & 78 & 78.4 & 78 & 77.4 & 77.4 & 77.4 & 77.4 & 77 & $77^{\# \$}$ \\
\hline BM24 & 35 & 91 & 94 & 89 & 84.1 & 80 & 81 & 82 & 79 & 85 & 79 & 85 & $79^{\# \$}$ \\
\hline BM25 & 36 & 94 & 87 & 79 & 76 & 76 & 75 & 73 & 75 & 77 & 71 & 71 & $71^{\# \$}$ \\
\hline BM26 & 38 & 91 & 101 & 97 & 85 & 82 & 80 & 80 & 80 & 80 & 80 & 80 & $80^{\#}$ \\
\hline BM27 & 40 & 91 & 100 & 109 & 85 & 85 & 84 & 85 & 84 & 84 & 85 & 84 & $84^{\# \$}$ \\
\hline
\end{tabular}

\& Solved by CPLEX 11.2;

* Proven optimal solution;

\# New solution compared to OGA;

${ }^{\$}$ New solution compared to IGA. 
Table 9. The best solutions (heights) of the new cases by different methods

\begin{tabular}{|c|c|c|c|c|c|c|c|c|c|c|c|c|}
\hline & & & & \multicolumn{2}{|c|}{ OGA } & \multicolumn{2}{|c|}{ IGA } & \multicolumn{5}{|c|}{ GSF } \\
\hline & $N$ & Rdm & MILP ${ }^{\&}$ & Rvs & Swp & Rvs & Swp & GGA1 & GGA2 & GGA3 & GGA4 & GGA5 \\
\hline SM00 & 10 & 80 & $68 *$ & 72 & 72 & 72 & 71 & 70 & 72 & 71 & 71 & $70^{\# \$}$ \\
\hline SM11 & 11 & 96 & 80 & 84 & 88 & 83 & 83 & 80 & 83 & 80 & 83 & $80^{\# \$}$ \\
\hline SM12 & 20 & 110 & 90 & 89 & 89 & 89 & 89 & 89 & 90 & 89 & 89 & $87^{\# \$}$ \\
\hline SM13 & 20 & 113 & 104 & 98 & 98 & 98 & 95 & 95 & 98 & 97 & 96 & $94^{\# \$}$ \\
\hline SM14 & 31 & 109 & 105 & 91 & 91 & 90 & 90 & 90 & 90 & 89 & 89 & $89^{\# \$}$ \\
\hline SM15 & 40 & 126 & 118 & 106 & 103 & 100 & 100 & 98 & 100 & 100 & 100 & $97^{\# \$}$ \\
\hline BM28 & 13 & 77.4 & 61 & 64 & 64 & 63.1 & 63.1 & 61 & 63.1 & 61 & 61 & $61^{\# \$}$ \\
\hline BM29 & 30 & 106 & 100 & 90 & 89 & 91 & 89 & 89.4 & 90 & 89.4 & 87.4 & $87.4^{\# \$}$ \\
\hline BM30 & 35 & 96 & 99 & 89 & 85 & 86 & 85 & 85 & 84 & 83 & 83 & $83^{\# \$}$ \\
\hline BM31 & 40 & 87 & 98 & 78 & 78 & 78 & 76 & 76 & 78 & 78 & 76 & $75^{\# \$}$ \\
\hline BM32 & 45 & 99 & 102 & 97 & 89 & 90 & 88 & 88 & 89 & 89 & 89 & $88^{\#}$ \\
\hline BM33 & 50 & 88 & 95 & 76 & 73 & 72 & 71 & 71 & 71 & 71 & 71 & $70^{\# \$}$ \\
\hline BM34 & 55 & 108 & 116 & 99 & 96 & 94 & 94 & 93 & 94 & 91 & 91 & $90^{\# \$}$ \\
\hline BM35 & 60 & 86 & 95 & 81 & 78 & 74 & 74 & 73 & 74 & 74 & 74 & $73^{\# \$}$ \\
\hline BM36 & 65 & 91 & 104 & 87 & 83 & 83 & 82 & 80 & 82 & 81 & 81 & $79^{\# \$}$ \\
\hline BM37 & 70 & 105 & 112 & 91 & 89 & 88 & 88 & 86 & 88 & 87 & 88 & $86^{\# \$}$ \\
\hline BM38 & 75 & 96 & 107 & 91 & 90 & 88 & 88 & 86 & 88 & 87 & 86 & $86^{\# \$}$ \\
\hline BM39 & 80 & 107 & 121 & 102 & 95 & 93 & 93 & 90 & 92 & 90 & 90 & $90^{\# \$}$ \\
\hline BM40 & 20 & 107.4 & 96 & 90 & 92 & 91 & 91 & 90 & 90 & 90 & 90 & $90^{\# \$}$ \\
\hline BM41 & 20 & 103.1 & 87 & 86 & 85.4 & 85 & 85 & 84.1 & 85.4 & 85 & 85.4 & $84.1^{\# \$}$ \\
\hline
\end{tabular}

\& Solved by CPLEX 11.2;

* Proven optimal solution;

\# New solution compared to OGA;

${ }^{\$}$ New solution compared to IGA.

\subsubsection{Comparison of Filled Rates $F_{1}$ and $F_{2}$}

Tables A-1 3 show the filled rates comparison of the methods. As defined in $\S 3.2 .3, F_{1}$ is calculated with respect to the net volume of the bin used, while $F_{2}$ is calculated with respect to the volume of the final cage bin, which reflects the actual degree of the packing tightness.

With the packing height $H$ available and given $L \times W$ of the bin, and the total volume of the packed cartons, $F_{1}$ can be calculated using Eq. 4 . But to calculate $F_{2}$ with Eq. $5, L_{c} \times W_{c}$ of the cage bin has to be given. For manual packing with only the height $H$, rather than the detailed solution with coordinates $(x, y, z)$, $L_{c} \times W_{c}$ is impossible, hence $F_{2}$ is not given for manual packing. For the solutions by MILP and OGA, a conversion program is designed to change the solutions into new format with $F_{1}, F_{2}$ and relative position. For the MILP model, it is found that most of them have the equal $F_{1}$ and $F_{2}$. This phenomenon is plausible from the constraints and the Big-M used in the MILP model. The results by the random search are to be compared with those by manual packing and MILP, thus without $F_{2}$ presented.

The cases studied can be categorized into five groups as listed in Table 10, where the old cases are divided into two groups, and the new cases into three groups. 
Table 10. Groups of the cases studied

\begin{tabular}{|c|c|c|c|c|}
\hline Group & Cartons $\boldsymbol{N}$ & Bin Type & Results & Old or New Cases \\
\hline 1 & $N \leq 26$ & small or big-bin & Tables 8, A-1, A-4 & old \\
\hline 2 & $27 \leq N \leq 40$ & big-bin & Tables 8, A-1, A-4 & old \\
\hline 3 & $10 \leq N \leq 40$ & $\begin{array}{c}\text { small-bin and } \\
\text { BM28, BM40, BM41 }\end{array}$ & Tables 9, A-2, A-5 & new \\
\hline 4 & $30 \leq N \leq 80$ & big-bin & Tables 9, A-2, A-5 & new \\
\hline 5 & $\begin{array}{c}10 \leq N \leq 20 \\
\text { Heterogeneous }\end{array}$ & small or big-bin & Tables 9, A-3, A-5 & new \\
\hline
\end{tabular}

The old cases in Table A-1 can be classified into two groups, group $1(N \leq 26)$ with a small number of cartons to be packed, group $2(27 \leq N \leq 40)$ with a moderate number of cartons.

In group $1(N \leq 26)$, a lower average $F_{1}(65.68 \%$ for small-bin packing and $65.71 \%$ for big-bin packing) by the manual operation for both small-bin packing and big-bin packing; as a contrast, the random search achieves a higher average $F_{1}(72.05 \%$ and $74.25 \%)$. In group 1, MILP model can obtain much higher average $F_{1}(81.99 \%$ and $81.06 \%)$ than the random search. The average $F_{1}$ by OGA (82.88\% and $82.61 \%)$ and IGA $(83.99 \%$ and $83.95 \%)$ increases a bit. The average $F_{1}$ by GGA5 (84.84\% and $84.64 \%$ ) increases about $2 \%$ against OGA. Fig. 13 shows the situation of the average $F_{1}$ for group 1, where it can be seen that MILP, OGA, IGA and GGA5 increase $F_{1}$ by $15 \% \sim 19 \%$ with respect to the manual packing.

In group $2(27 \leq N \leq 40)$, the packing by the manual operation, the random search and MILP model has similar lower average $F_{1}(74.31 \%, 74.48 \%$ and $77.73 \%$ respectively); but OGA, IGA and GGA5 increase by $14 \% \sim 16 \%$ with respect to the manual packing. For these moderate-size instances, our random search obtains almost the same average $F_{1}$ as the manual packing. That is why the packing solutions by the random search for the new cases can be regarded as the real manual packing solutions. It should be noticed is that GGA5 achieves up to an average $F_{1}$ of $90 \%$. Fig. 14 shows the situation of the average $F_{1}$ for group 2. It is obvious that GA-based methods take great advantage over the others.

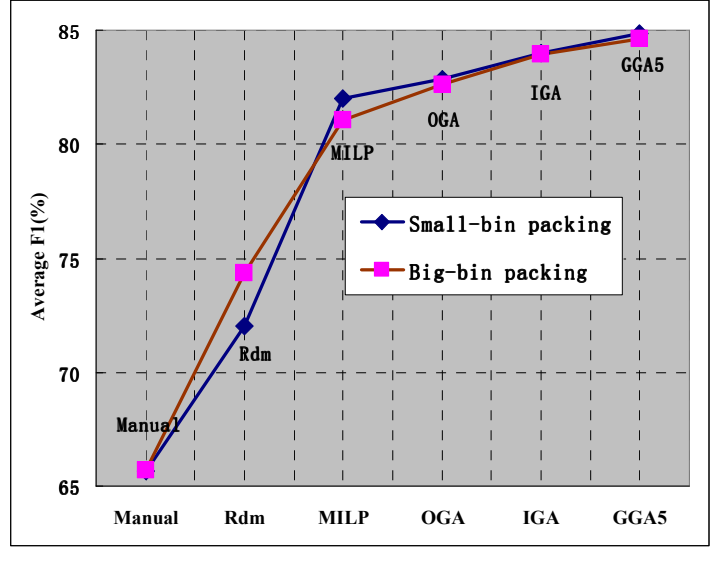

Fig. 13 Average $F_{1}$ for small-size old cases (group 1)

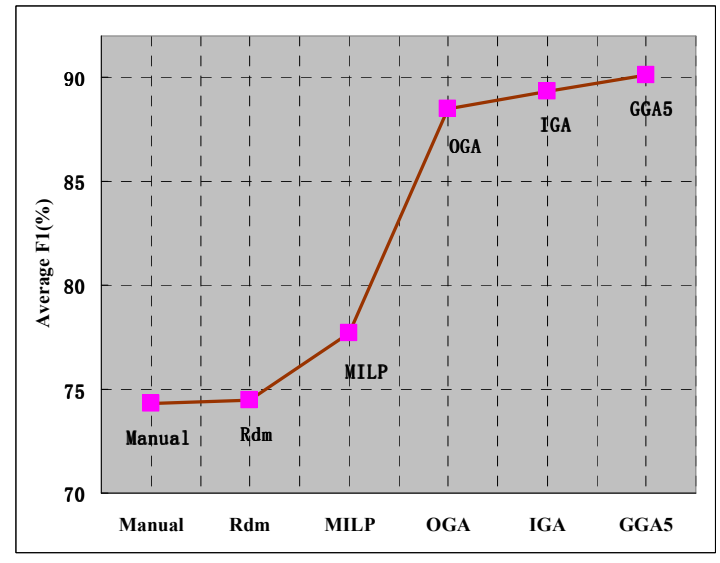

Fig. 14 Average $F_{1}$ for moderate-size old cases (group 2) 


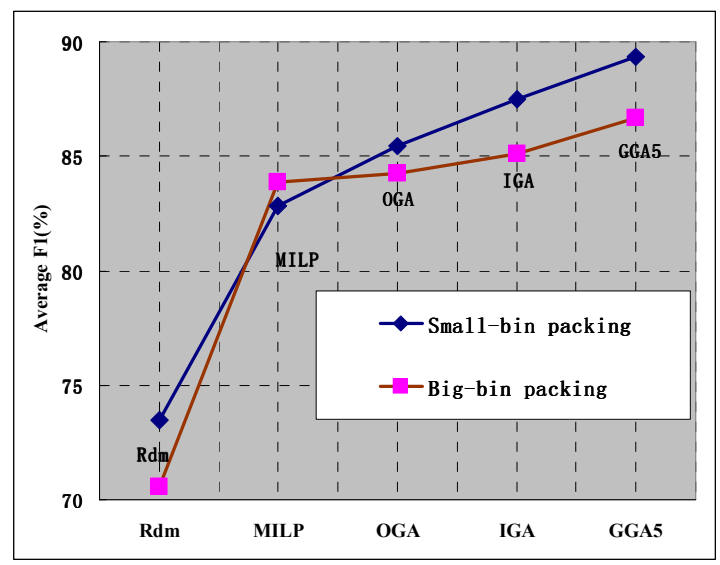

Fig. 15 Average $F_{1}$ for small-size new cases (group 3)

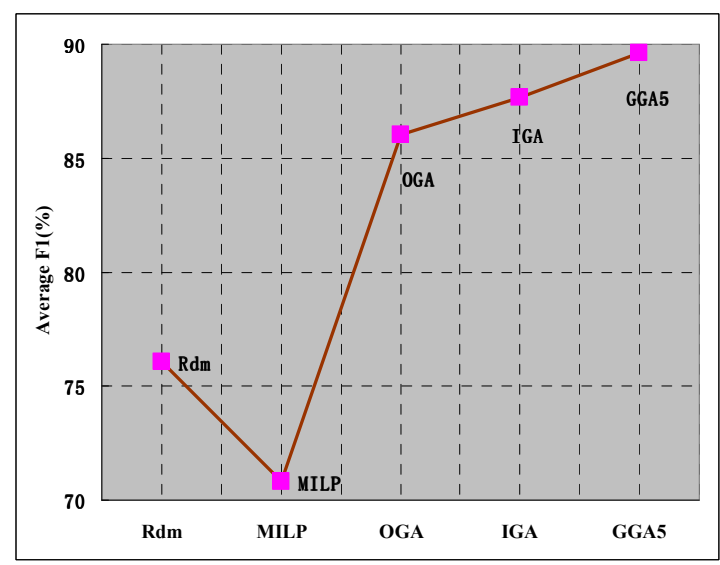

Fig. 16 Average $F_{1}$ for large-size new cases (group 4)

The new cases in Table A-2 can also be classified into two groups: the small-bin packing cases $(10 \leq N \leq 40)$ and the big-bin cases (BM28, BM40 and BM41) with more heterogeneous cartons $(13 \leq N \leq 20)$ are put into group 3 (small-size instances in Table A-2), the big-bin packing cases (BM29 39) with a large number of cartons are put into group 4 (30 $\leq N \leq 80$ in Table A-2, big-bin packing).

In group 3, the situation of the average $F_{1}$ is similar to group 1, it is also can be seen that MILP, OGA, IGA and GGA5 achieve higher average $F_{1}(83 \% \sim 90 \%$, see Fig. 15).

The situation of group 4 varies: the MILP model's performance declines severely. For this group of large-size instances, OGA, IGA and GGA5 demonstrate distinct advantage over the other techniques, as shown in Fig. 16.

Table A-3 provides statistic results of the heterogeneous cases in which each carton belongs to a different carton type. These cases are put into group 5 (with $10 \leq N \leq 20$ different cartons). For this group, MILP shows its dominance with higher average $F_{1}$ over OGA and IGA. However, GGA5 is still the best! Fig. 17 shows the situation of the average $F_{1}$ for group 5.

From group 1 to group 5, IGA obtains higher average

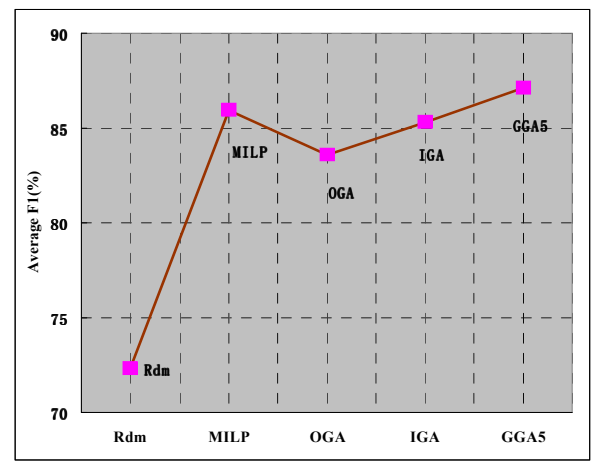

Fig. 17 Average $F_{1}$ for heterogeneous group 5 $F_{1}$ than OGA, and GGA5 higher than IGA logically and rationally. Another observation worth noticing is that, when OGA and IGA obtain the same packing height $(H)$ for a packing case, thus the same value of $F_{1}$, but IGA achieves higher value of $F_{2}$ than OGA (see the cases SM01, 07 and 10, BM02, 06, 08, 10, 12, 14, 19 22, 27, 29 and 30, totally 16 cases). That means IGA pack cartons much tighter than OGA owing to the improved decoding procedure, especially because of index 2 and new parallel moving strategy adopted. 


\subsubsection{Comparison of Computational Time}

Computational time is an important factor to evaluate an algorithm. Tables A-4 and A-5 present the computational times $(C T)$ of five methods in solving the old and new instances, and the time is in seconds. From these two tables, we can see the following facts:

(1) The random search based on the decoding procedure can be finished within very short time $\left(C T_{R}<3 \mathrm{~s}\right)$. So the decoding procedure can be used for packing simulation experiments conveniently.

(2) For most cases, MILP model reach the limited running time, 2 hours (7200s). Taking the volume utilization into account, MILP model can only find satisfactory solutions for the small-size instances in groups 1, $\mathbf{3}$ and $\mathbf{5}$ within the limited time.

(3) The solution speed of IGA is about 14 times faster than that of OGA. From the comparison of filled rates, IGA achieves higher filled rates $\left(F_{1}\right.$ and $\left.F_{2}\right)$ than OGA.

(4) The solution speed of GGA5 is about 4 times faster than that of OGA. What is more, GGA5 improves the average $F_{1}$ by approximate $2 \sim 4 \%$ with respect to OGA. When OGA is applied to the large-size instances BM37, 38 and 39, the solution times are 8437 seconds ( 2 hours and 20 minutes), 11189 seconds (about 3 hours), 14601 seconds (about 4 hours) respectively, all over 2 hours. But for these three cases, GGA5 has completed the search within 2016 seconds (about 33 minutes).

(5) The average number of global iterations $g$ in GGA5 is about 4 (3.84 for the old cases, 4.45 for the new cases). For the cases BM08, BM24 and SM15, the global iterations $g$ of GGA5 are 6, 9 and 7 respectively. These data reveal the fact that the global iterations of GGA5 are indeed essentially necessary to achieve the high quality solutions.

\subsubsection{Comprehensive Comparison}

From the comparison and analysis of the packing height $(H)$, filled rates $\left(F_{1}\right.$ and $\left.F_{2}\right)$ and computational time $(C T)$, the following comprehensive recognitions can be drawn out:

(1) The random search has an equal or even higher average $F_{1}$ than the manual packing, with very short search time (less than 3 seconds). This means that the decoding procedure is effective in simulating a packing solution.

(2) MILP model can only obtain satisfactory solutions for the small-size instances $(N \leq 26)$ within acceptable time (e.g. 2 hours); but for moderate-size or large-size instances, the exact model, which is limited by the current computing resources, such as computational time, memory and CPU speed, shows its apparent inferior position in contrast to the GA-based methods.

(3) OGA is indeed effective and efficient in solving the original small- and moderate-size instances, but has still left a larger space for further improvement. For the new cases including more heterogeneous cases, OGA shows its drawbacks in terms of solution speed and quality. As a contrast, IGA witnesses improvement not only on solution speed but also on solution quality.

(4) The novel GSF (GGA5) has obtained the best solutions for most of the old and new cases 
among the methods tested, within acceptable time (less than 33 minutes), hence is the best method tested in our work.

It should also be mentioned that in Wu et al. (2010), the achieved bin height for each test case was fed into the container loading algorithm provided by Martello et al. (2007) for runs of two hours. In most cases, the Martello et al. (2007) code was not able to provide a solution to load all the cartons within the given height limit. Since the results provided in this paper are better than those of Wu et al. (2010), it can be reasonably concluded that it would be even more difficult for the algorithm described in the Martello et al. (2007) to provide feasible results under the same settings in Wu et al. (2010).

\subsection{Tests on 3D-SPP Benchmark Instances}

\subsubsection{Result Comparison}

As explained earlier, the problem proposed in this paper is similar to the 3D-SPP but has some distinct features. For heuristics algorithms, it is common to exploit the problem features to ensure effectiveness and efficiency. Although the proposed algorithms did not take 3D-SPP into consideration at the design phase, one of the algorithms, GGA5, is applied to testify its effectiveness on some of the strip packing instances from Bischoff and Ratcliff (1995) as an additional way for benchmarking. We have to mention that the use of genetic algorithms actually has some challenges when the number of cartons to be packed increases and therefore the tests on the 3D-SPP benchmarking instances can be viewed as a 'stretch' for the current algorithms. We first apply the GGA5 to the selected 3D-SPP cases without imposing the orientation constraints (marked as 'w/o orient. con.') and subsequently consider the orientation constraints (marked as 'with orient. con.') in both generating chromosomes and mutating according to the specified orientation constraints, where the GA parameters remain the same, (popsize, $\left.C_{r}, M_{r}\right)=(400,0.7,0.3)$. We then compare the results with those presented in Bortfeldt and Mack (2007) and Allen et al. (2011) where some of the state of the art results are available. We also notice that a working paper by Bortfeldt and Jungmann (2010) has seemingly improved the results significantly. Table 11 shows the volume utilization $\left(F_{1}\right)$ for all these methods.

For GGA5, most of the cases where the orientation constraints are not imposed generate slightly better results than those with orientation constraints imposed, except for BR03 and BR10. Look across GGA5 with orientation constraints, Bortfoldt and Mark (2007) and Allen et al. (2011), the volume utilizations achieved by GGA5 for BR01 03 and BR05 are higher than those obtained by Bortfeldt and Mack (2007) and Allen et al. (2011). We also get close results for the rest cases. In terms of overall average volume utilization, ours is also higher than that of Bortfeldt and Mack (2007) and Allen et al. (2011). Computational time wise, ours is longer than that of Bortfeldt and Mack (2007) and Allen et al. (2011) due to operations involved in the genetic algorithms. The results from Bortfeldt and Jungmann (2010), where the algorithm tries to solve a series of container loading problems iteratively (in reduced length), seem to be the best among all methods presented in Table 11. 
Table 11. Results for the test cases BR01 10 from Bischoff and Ratcliff (1995)

\begin{tabular}{|c|c|c|c|c|c|c|c|}
\hline \multirow{2}{*}{} & \multirow{2}{*}{ Types } & \multirow{2}{*}{$\boldsymbol{N}$} & \multicolumn{2}{|c|}{ GGA5 $\boldsymbol{F}_{\mathbf{1}}(\mathbf{\%})$} & $\begin{array}{c}\text { B\&M (2007) } \\
\boldsymbol{F}_{\mathbf{1}}(\mathbf{\%})\end{array}$ & $\begin{array}{c}\text { Allen et al. (2011) } \\
\boldsymbol{F}_{\mathbf{1}}(\mathbf{\%})\end{array}$ & $\begin{array}{c}\text { B\&J (2010) } \\
\boldsymbol{F}_{\mathbf{1}}(\mathbf{\%})\end{array}$ \\
\hline BR01 & 3 & 139.4 & $\mathbf{9 1 . 7 0}$ & $\mathbf{9 1 . 5 2}$ & 87.3 & 90.0 & 94.2 \\
\hline BR02 & 5 & 140.1 & $\mathbf{9 1 . 8 5}$ & $\mathbf{9 0 . 8 4}$ & 88.6 & 89.6 & 94.8 \\
\hline BR03 & 8 & 135.4 & $\mathbf{9 0 . 5 3}$ & $\mathbf{9 0 . 5 3}$ & 89.4 & 89.9 & 94.7 \\
\hline BR04 & 10 & 132.2 & $\mathbf{9 0 . 3 1}$ & 89.49 & $\mathbf{9 0 . 1}$ & 88.8 & 94.2 \\
\hline BR05 & 12 & 127.8 & $\mathbf{8 9 . 9 1}$ & $\mathbf{8 9 . 5 7}$ & 89.3 & 88.5 & 93.9 \\
\hline BR06 & 15 & 133.8 & $\mathbf{8 9 . 7 5}$ & 89.47 & $\mathbf{8 9 . 7}$ & 88.6 & 93.5 \\
\hline BR07 & 20 & 129.2 & 88.59 & 88.23 & $\mathbf{8 9 . 2}$ & 88.7 & 92.6 \\
\hline BR08 & 30 & 138.0 & 87.44 & 87.32 & 87.9 & $\mathbf{8 8 . 3}$ & 91.8 \\
\hline BR09 & 40 & 127.5 & 86.54 & 86.19 & 87.3 & $\mathbf{8 7 . 9}$ & 91.5 \\
\hline BR10 & 50 & 129.2 & 86.30 & 86.41 & 87.6 & $\mathbf{8 7 . 9}$ & 91.3 \\
\hline Average & 19.3 & 133.3 & $\mathbf{8 9 . 2 9}$ & $\mathbf{8 8 . 9 6}$ & 88.64 & 88.82 & 93.2 \\
\hline
\end{tabular}

\subsubsection{Difference Analysis}

In terms of measuring difficulty of packing problems, Smith-Miles and Lopes (2012) review some common combinatorial optimization problems. One particular factor proposed for bin packing in that paper is the average carton volume against the bin volume as one of the measurements for difficulty. Fig. 18 presents the carton volume against the bin volume for each carton in cases of SM14, BM31, BM39, BR06-008 and BR10-009. It can be observed that in terms of number of cartons for packing, SM13 and BM34 (and most other cases investigated in this paper) represent a category of problem with relatively small number of cartons but significantly differ in terms of carton sizes and number of cartons available per carton type. On the contrary, BR06-008 and BR10-009 represent typical strip packing problems, where more cartons are to be packed and each carton type usually includes quite a number of cartons; moreover, the size difference between types is relatively lower. These features were effectively utilized by the algorithms proposed in Bortfeldt and Mack (2007) and Allen et al. (2011), and other algorithms with effective layer-packing and strip building.

Fig. 19 further indicates the difference between our investigated cases and those of traditional 3D-SPP, where the maximum, minimum and average carton volumes against the 'bin' (container) volume are presented. It can be observed that the maximum cartons in some selected cases from this paper have a volume that is more than $20 \%$ of the 'bin' itself; most of the maximum cartons are more than $10 \%$ of the 'bin' volume, while none of the cases selected from BR01 to BR10 has a carton volume more than $3 \%$ of the overall container volume. 


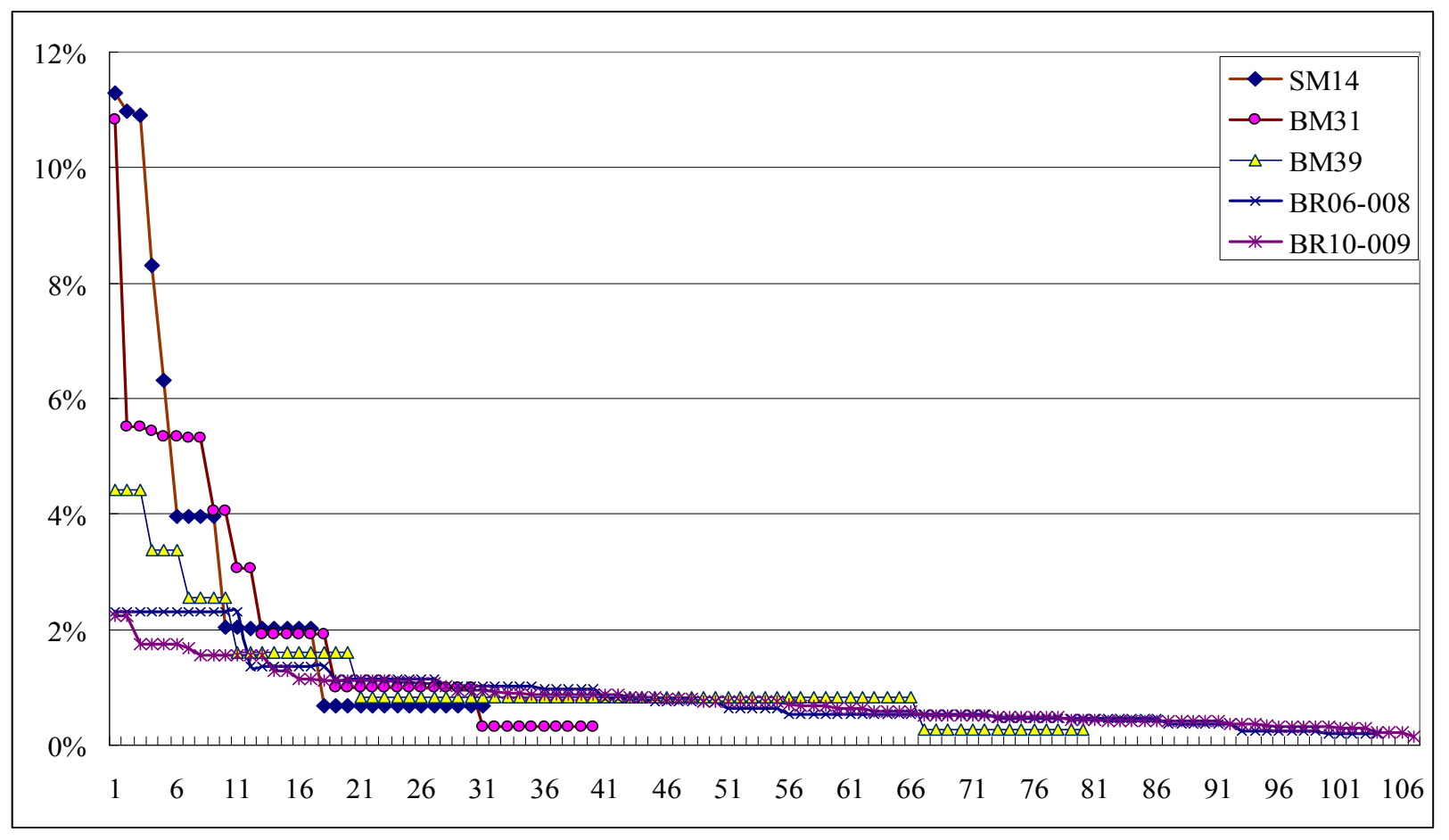

Fig. 18 Carton vs. bin volume comparison for SM14, BM31, BM39, BR06-008 and BR10-009

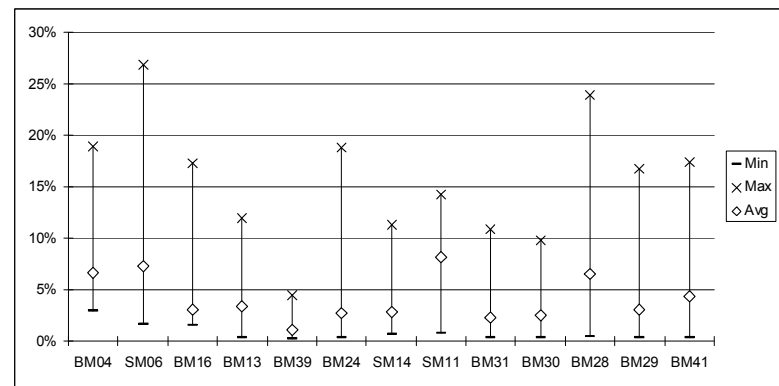

(a) Cases from this paper

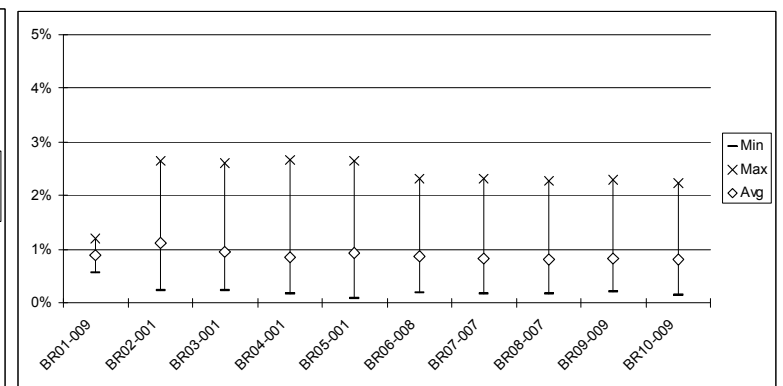

(b) Cases from BR01 to BR10

Fig. 19 Maximum, minimum and average volume against ‘bin' volume

\section{Conclusion}

This study, with the aim to solve the challenging real-world 3D packing problem with variable carton orientations, has made meticulous improvement to the packing (decoding) procedure, devised a particular genetic algorithm with customized selection, crossover and mutation operators, and adopted a novel global search framework based on the concept of evolutionary gradient. The numerical experiments indicate that the selected scenario of the global search framework is able to solve the investigated packing problem effectively and efficiently. The novelty and contribution of this work can be summarized as follows:

(1) A novel index (index2) is defined for the selection of reference points in locating a carton in the bin, which is the crucial reason that makes the packing more compact.

(2) A new parallel moving strategy is proposed to reduce possible gaps and to avoid apparently 
invalid moves thus save computational time and provide faster and more compact packing.

(3) The relative positions which indicate the cartons that are touched on the left, in front and at the bottom of a particular carton can be reported via the improved decoding procedure. This, to our knowledge, is the first attempt to presenting relative positions, which provides to be very convenient and handy to guide manual packing in practice.

(4) Appropriate genetic components have been selected and well combined together with simultaneous consideration of solution speed and precision.

(5) The concept of evolutionary gradient has been proposed considering the usual existing diversity of the solutions obtained by meta-heuristics, e.g. the genetic algorithm. The global search framework has subsequently been devised in order to make full use of the solution ability of the selected genetic algorithm.

(6) Several scenarios of the GSF have been constructed and examined with the purpose to improve the solution speed. A suitable scenario (GGA5) has then been selected and tested with a wide range of packing cases, including larger-size and more heterogeneous instances. For most of the instances, the GGA5 has achieved better solutions than OGA, and the computational times are much shorter.

With the above novelty, the GSF is able to achieve packing solutions with average volume utilization up to $\mathbf{8 5} \mathbf{9 0 \%}$ within half an hour; for some cases, the volume utilization reaches 92 95\%. In contrast, the manual packing only has average volume utilization less than $\mathbf{7 5 \%}$, with very few exceptional cases reaching $80 \sim 82 \%$. The average increment of volume utilization by GGA5 can be more than $15 \%$ against the current practical operations.

To sum up, the GSF can provide high-fill-rate packing solutions with information of the relative positions, orientations and coordinates of the cartons. Furthermore, with the ability to handle arbitrary orientations and heterogeneous cartons, the proposed method can be easily tailored and applied to other types of bin packing problems.

The GSF can be easily transformed into a synchronous parallel version, because the synchronization and communication, which are the difficult issues in parallel computing, are already available. The further interesting work is to design an asynchronous parallel version of this framework.

\section{Acknowledgement}

The authors would like to acknowledge Emerson Singapore Distribution Center for providing the operational packing data, and give our special thanks to Mr. Jack Wong and Mr. Andy Chang.

The authors would also like to thank the anonymous reviewers for their constructive and pertinent comments, which have significantly improved the paper into its current form. 


\section{References}

Allen, S. D., Burke, E. K. and Kendall, G. 2011. A hybrid placement strategy for the threedimensional strip packing problem. European Journal of Operational Research, 209, 219-227.

Baltacioglu, E., Moore, J. T., Hill Jr., R. R. 2006. The distributor's three dimensional pallet-packing problem: a human intelligence-based heuristic approach. International Journal of Operational Research 1, 249-266.

Bean, J. 1994. Genetic algorithms and random keys for sequencing and optimization. ORSA Journal on Computing 6, 154-60.

Bortfeldt, A. and Jungmann, S., 2010. A tree search algorithm for solving the multi-dimensional strip packing problem with guillotine cutting constraint. Working paper. Accessed on 01 October 2011, URL: ftp://ftp.fernuni-hagen.de/pub/fachb/wiwi/winf/forschng/publi/DBFakWiWi458.pdf

Bortfeldt, A. and Mack, D., 2007. A heuristic for the three-dimensional strip packing problem. European Journal of Operational Research, 183, 1267-1279.

Bischoff, E. E., Janetz, F., Ratcliff, M. S. W. 1995. Loading pallets with non-identical items. European Journal of Operational Research 84, 681-692.

Bischoff, E. E., Marriott, M. D. 1990. A comparative evaluation of heuristics for container loading. European Journal of Operational Research 44, 267-276.

Bischoff, E. E., Ratcliff, M. S. W. 1995. Issues in the development of approaches to container loading. OMEGA - International Journal of Management Science 23, 377-90.

Bortfeldt, A. , Gehring, H. 1998. Applying tabu search to container loading problems. In: Operations research proceedings. Berlin: Springer Press, 533-538.

Bortfeldt, A., Gehring, H. 2001. A hybrid generic algorithm for the container loading problem. European Journal of Operational Research 131, 143-161.

Ceselli, A., Righini, G. 2008. An optimization algorithm for the ordered open-end bin-packing problem. Operations Research 36, 425-436.

Chen, C. S., S. M. Lee, Q. S. Shen. 1995. An analytical model for the container loading problem. European Journal of Operational Research 80, 68-76.

Crainic, T. G., Perboli, G., Tadei, R. 2008a. TS²PACK: A two-level tabu search for the threedimensional bin packing problem. European Journal of Operational Research. In press.

Crainic, T. G., Perboli, G., Tadei, R. 2008b. Extreme point-based heuristics for three- dimensional bin packing. INFORMS Journal on Computing 20, 368-384

den Boef, E., J. Korst, S. Martello, D. Pisinger, D. Vigo. 2005. Erratum to "The three-dimensional bin packing problem": Robot-packable and orthogonal variants of packing problems. Operations Research 53, 735-736.

Dosa, G. 2007. The tight bound of first fit decreasing bin-packing algorithm is FFD(I) $\leq 11 / 9 \mathrm{OPT}(\mathrm{I})+6 / 9$. In: Combinatorics, Algorithms, Probabilistic and Experimental Methodologies, volume 4614 of LNCS, pages 1-11. Springer, Berlin.

Egeblad, J., Pisinger, D. 2009. Heuristic approaches for the two- and three- dimensional knapsack packing problem. Computers \& Operations Research 36, 1026-1049.

Fanslau, T. and Bortfeldt, A. 2010. A tree search algorithm for solving the container loading problem. INFORMS Journal on Computing, 22, 222-235.

Faroe, O., Pisinger, D., Zachariasen, M. 2003. Guided local search for three- dimensional bin-packing 
problem. INFORMS Journal on Computing 15, 267-283.

Fekete, S. P., Schepers, J., van der Veen, J. C. 2007. An exact algorithm for higher- dimensional orthogonal packing. Operations Research 55, 569-587.

Gehring, H., Bortfeldt, A. 1997. A genetic algorithm for solving the container loading problem. International Transactions in Operational Research 4, 401-418.

Gehring, H., Bortfeldt, A. 2002. A parallel genetic algorithm for solving the container loading problem. International Transactions in Operational Research 9, 497-511.

Gen, M., Cheng, R. 1997.Genetic Algorithms and Engineering Optimization. New York: Wiley.

George, J. A., Robinson, D. F. 1980. A heuristic for packing boxes into a container. Computers and Operations Research, 7 147-156.

Goldberg, D. E. 1989. Genetic Algorithms in Search, Optimization and Machine Learning. Reading, MA: Addison-Wesley.

Goldberg, D. E., Deb, K. 1991. A comparative analysis of selection schemes used in genetic algorithms. In: Rawlins, G., (Eds), Foundations of Genetic Algorithms, Morgan Kaufmann.

Goncalvez, J. F. and Resende, M. C. 2012. A parallel multi-population biased random-key genetic algorithm for a container loading problem. Computers \& Operations Research, 39, 179-190.

He, Y. 2007. Research on Meta-Heuristic Methods for Large-Scale Complex Scheduling in Process Industry. Ph.D Dissertation, Hong Kong University of Science and Technology.

He, Y., C. W. Hui. 2008. A rule-based genetic algorithm for the scheduling of single-stage multi-product batch plants with parallel units. Computers and Chemical Engineering 32, 3067-3083.

He, Y., Hui, C. W. 2009. Meta-Heuristics for Large-Scale Process Scheduling. VDM Verlag, Saarbrücken, Germany.

He, Y., Hui, C. W. 2010. A novel search framework for multi-stage process scheduling with tight due dates. AIChE Journal 56, 2103-2121.

Huang, W., He, K. 2009. A new heuristic algorithm for cuboids packing with no orientation constraints. Computers \& Operations Research 36, 425-432.

Lim, A., Rodrigues, B., Yang, Y. 2005. 3-D Container Packing Heuristics. Applied Intelligence 22, $125-134$.

Lodi, A., Martello, S., Vigo, D. 2002. Heuristic algorithms for the three-dimensional bin packing problem. European Journal of Operational Research 141, 410-420.

Loh, T. H., Nee, A. Y. C. 1992. A packing algorithm for hexahedral boxes. In: Proceedings of the Conference of Industrial Automation, Singapore, 115-126.

Martello, S., Pisinger, D., Vigo, D., 2000. The three-dimensional bin packing problem. Operations Research 48 (2), 256-267.

Martello, S., Pisinger, D., Vigo, D., den Boef, E., Korst, J. 2007. Algorithm 864: General and robot-packable variants of the three-dimensional bin packing problem. ACM Transactions on Mathematical Software 33, 1-12.

Pisinger, D. 2002. Heuristics for the container loading problem. European Journal of Operational Research 141, 382-392.

Poon, P. W., Carter, J. N. 1995. Genetic algorithm crossover operators for ordering applications. Computers \& Operations Research 22, 135-47. 
Smith-Miles, K. and Lopes, L. 2012. Measuring instance difficulty for combinatorial optimization problems. Computers \& Operations Research, 39, 875-889.

Wäscher, G., Hauber, H. and Schumann, H. 2007. An improved typology of cutting and packing problems. European Journal of Operational Research, 183, 1109-1130.

Wu, Y., Li, W., Goh, M., de Souza, R. 2010. Three dimensional bin packing problem with variable bin height. European Journal of Operational Research 202, 347-355.

Yang, J., Leung, J. Y. T. 2003. The ordered open-end bin-packing problem. Operations Research 51, $759-770$.

Zhang, D., Wei, L., Chen, Q., Chen, H. 2007. A combinatorial heuristic algorithm for the three-dimensional packing problem (in Chinese, with English abstract). Journal of Software 18, 2083-2089. 
Appendix A: Solution Results by Different Methods

Table A-1. Comparison of filled rates of the old cases by different methods

\begin{tabular}{|c|c|c|c|c|c|c|c|c|c|c|c|c|c|c|c|c|}
\hline & & & anual & & $\mathrm{dm}$ & $\mathbf{M}$ & ILP & & GA(Sv & p) & & GA(Sw & & & GGA5 & \\
\hline & $N$ & $H$ & $F_{1}(\%)$ & $H$ & $F_{1}(\%)$ & $H$ & $F_{1}(\%)$ & $H$ & $F_{1}(\%)$ & $F_{2}(\%)$ & $H$ & $F_{1}(\%)$ & $F_{2}(\%)$ & $H$ & $F_{1}(\%)$ & $F_{2}(\%)$ \\
\hline SM01 & 8 & 91 & 69.13 & 92 & 68.38 & 80 & 78.64 & 80 & 78.64 & 83.00 & 80 & 78.64 & 87.49 & 80 & 78.64 & 87.49 \\
\hline SM02 & 8 & 56 & 54.66 & 40 & 76.52 & 37 & 82.73 & 37 & 82.73 & 82.73 & 37 & 82.73 & 82.73 & 37 & 82.73 & 82.73 \\
\hline SM03 & 9 & 69 & 57.25 & 53 & 74.54 & 47 & 84.05 & 47 & 84.05 & 84.05 & 47 & 84.05 & 84.05 & 47 & 84.05 & 84.05 \\
\hline SM04 & 9 & 91 & 78.33 & 103 & 69.20 & 82 & 86.93 & 83 & 85.88 & 85.88 & 83 & 85.88 & 85.88 & 82 & 86.93 & 86.93 \\
\hline SM05 & 10 & 56 & 80.91 & 61 & 74.28 & 52 & 87.13 & 52 & 87.13 & 90.53 & 52 & 87.13 & 90.53 & 52 & 87.13 & 90.53 \\
\hline SM06 & 12 & 53 & 62.42 & 48 & 68.93 & 38 & 87.06 & 40 & 82.71 & 84.19 & 40 & 82.71 & 84.19 & 38 & 87.06 & 87.06 \\
\hline SM07 & 17 & 70 & 64.24 & 62 & 72.53 & 62 & 72.53 & 62 & 72.53 & 80.32 & 62 & 72.53 & 88.84 & 62 & 72.53 & 88.84 \\
\hline SM08 & 17 & 91 & 63.74 & 77 & 75.33 & 71 & 81.70 & 69 & 84.06 & 85.56 & 62 & 93.55 & 93.55 & 62 & 93.55 & 93.55 \\
\hline SM09 & 19 & 71 & 60.73 & 62 & 69.55 & 62 & 69.55 & 52 & 82.92 & 82.92 & 51 & 84.55 & 84.55 & 50 & 86.24 & 86.24 \\
\hline SM10 & 21 & 85 & 65.34 & 78 & 71.20 & 62 & 89.57 & 63 & 88.15 & 89.30 & 63 & 88.15 & 91.36 & 62 & 89.57 & 89.57 \\
\hline Avg01 10 & & & 65.68 & & 72.05 & & 81.99 & & 82.88 & 84.85 & & 83.99 & 87.32 & & 84.84 & 87.70 \\
\hline Increase & & & & & 6.37 & & 16.31 & & 17.21 & & & 18.32 & & & 19.17 & \\
\hline BM01 & 5 & 91 & 68.61 & 84.1 & 74.24 & 84.1 & 74.24 & 84.1 & 74.24 & 80.15 & 84.1 & 74.24 & 80.15 & 84.1 & 74.24 & 80.15 \\
\hline BM02 & 11 & 91 & 77.92 & 99 & 71.63 & 83.1 & 85.33 & 84.1 & 84.32 & 85.65 & 84.1 & 84.32 & 88.78 & 84.1 & 84.32 & 89.56 \\
\hline BM03 & 12 & 45 & 63.47 & 47 & 60.77 & 32 & 89.25 & 32 & 89.25 & 92.50 & 32 & 89.25 & 92.50 & 32 & 89.25 & 92.50 \\
\hline BM04 & 13 & 91 & 72.92 & 98 & 67.71 & 77.4 & 85.73 & 83.4 & 79.57 & 83.71 & 77.4 & 85.73 & 87.92 & 77.4 & 85.73 & 87.92 \\
\hline BM05 & 15 & 91 & 72.23 & 90 & 73.03 & 79 & 83.20 & 79 & 83.20 & 89.53 & 79 & 83.20 & 89.53 & 78 & 84.27 & 89.74 \\
\hline BM06 & 16 & 67 & 48.62 & 47 & 69.31 & 40 & 81.44 & 40 & 81.44 & 81.79 & 40 & 81.44 & 82.65 & 40 & 81.44 & 83.52 \\
\hline BM07 & 21 & 48 & 63.56 & 40 & 76.27 & 40 & 76.27 & 40 & 76.27 & 78.76 & 38 & 80.29 & 86.95 & 37 & 82.46 & 84.55 \\
\hline BM08 & 22 & 59 & 70.44 & 54 & 76.97 & 53 & 78.42 & 51 & 81.49 & 83.28 & 51 & 81.49 & 85.03 & 48 & 86.59 & 86.96 \\
\hline BM09 & 23 & 94 & 66.64 & 80 & 78.30 & 82 & 76.39 & 72 & 87.00 & 88.29 & 71 & 88.23 & 90.31 & 71 & 88.23 & 90.31 \\
\hline BM10 & 23 & 91 & 58.11 & 62 & 85.29 & 63.1 & 83.80 & 61 & 86.68 & 86.76 & 61 & 86.68 & 87.89 & 61 & 86.68 & 88.73 \\
\hline BM11 & 25 & 91 & 72.08 & 80.1 & 81.89 & 78 & 84.10 & 77.4 & 84.75 & 85.78 & 73.4 & 89.37 & 92.54 & 73.4 & 89.37 & 92.54 \\
\hline BM12 & 26 & 94 & 53.93 & 66 & 76.80 & 68 & 74.54 & 61 & 83.10 & 84.33 & 61 & 83.10 & 85.21 & 61 & 83.10 & 85.96 \\
\hline Avg01 12 & & & 65.71 & & 74.35 & & 81.06 & & 82.61 & 85.04 & & 83.95 & 87.46 & & 84.64 & 87.70 \\
\hline Increase & & & & & 8.64 & & 15.35 & & 16.90 & & & 18.23 & & & 18.93 & \\
\hline BM13 & 27 & 83 & 73.88 & 85 & 72.14 & 78 & 78.62 & 71 & 86.37 & 87.50 & 69 & 88.87 & 89.26 & 68 & 90.18 & 91.51 \\
\hline BM14 & 28 & 95 & 74.42 & 94 & 75.22 & 88.4 & 79.98 & 78.4 & 90.18 & 90.49 & 78.4 & 90.18 & 91.06 & 78.4 & 90.18 & 91.06 \\
\hline BM15 & 29 & 91 & 71.86 & 94 & 65.97 & 87 & 75.17 & 75 & 87.20 & 88.33 & 74 & 88.37 & 88.76 & 73 & 89.58 & 90.91 \\
\hline BM16 & 29 & 63 & 65.29 & 54 & 76.17 & 52 & 79.10 & 48 & 85.69 & 86.81 & 47 & 87.51 & 89.43 & 47 & 87.51 & 89.43 \\
\hline BM17 & 29 & 91 & 79.13 & 101 & 71.30 & 88.4 & 81.46 & 80 & 90.01 & 91.03 & 78.4 & 91.85 & 93.12 & 78.4 & 91.85 & 93.16 \\
\hline BM18 & 30 & 70 & 80.17 & 70 & 80.17 & 73.4 & 76.45 & 63.1 & 88.93 & 89.01 & 63 & 89.07 & 89.94 & 63 & 89.07 & 89.94 \\
\hline BM19 & 30 & 94 & 60.11 & 78.4 & 72.07 & 79 & 71.52 & 63.1 & 89.54 & 90.18 & 63.1 & 89.54 & 90.49 & 63 & 89.69 & 90.07 \\
\hline BM20 & 30 & 94 & 74.56 & 94 & 74.56 & 87 & 80.56 & 79 & 88.71 & 89.10 & 79 & 88.71 & 90.81 & 78 & 89.85 & 91.18 \\
\hline BM21 & 30 & 95 & 73.95 & 94 & 74.74 & 87 & 80.75 & 78.4 & 89.61 & 90.48 & 78.4 & 89.61 & 90.93 & 78.4 & 89.61 & 92.20 \\
\hline BM22 & 31 & 91 & 73.93 & 85 & 79.15 & 87 & 77.33 & 78 & 86.26 & 86.63 & 78 & 86.26 & 88.15 & 77 & 87.38 & 90.38 \\
\hline BM23 & 33 & 95 & 74.14 & 94 & 74.93 & 93.4 & 75.41 & 78.4 & 89.84 & 90.15 & 77.4 & 91.00 & 91.39 & 77 & 91.47 & 92.66 \\
\hline BM24 & 35 & 91 & 81.83 & 94 & 79.22 & 89 & 83.67 & 80 & 93.08 & 93.40 & 82 & 90.81 & 91.69 & 79 & 94.26 & 95.18 \\
\hline BM25 & 36 & 94 & 68.72 & 87 & 74.25 & 79 & 81.76 & 76 & 84.99 & 85.36 & 73 & 88.48 & 88.87 & 71 & 90.98 & 92.32 \\
\hline BM26 & 38 & 91 & 80.81 & 101 & 72.81 & 97 & 75.81 & 82 & 89.68 & 90.07 & 80 & 91.92 & 93.12 & 80 & 91.92 & 93.12 \\
\hline BM27 & 40 & 91 & 81.81 & 100 & 74.44 & 109 & 68.30 & 85 & 87.58 & 87.96 & 85 & 87.58 & 88.87 & 84 & 88.62 & 89.93 \\
\hline Avg13 27 & & & 74.31 & & 74.48 & & 77.73 & & 88.51 & 89.10 & & 89.32 & 90.39 & & 90.14 & 91.54 \\
\hline Increase & & & & & 0.17 & & 3.42 & & 14.20 & & & 15.01 & & & 15.84 & \\
\hline
\end{tabular}


Table A-2. Comparison of filled rates of the new cases by different methods

\begin{tabular}{|c|c|c|c|c|c|c|c|c|c|c|c|c|c|c|}
\hline & \multirow[b]{2}{*}{$N$} & \multicolumn{2}{|c|}{ Rdm } & \multicolumn{2}{|c|}{ MILP } & \multicolumn{3}{|c|}{ OGA(Swp) } & \multicolumn{3}{|c|}{ IGA(Swp) } & \multicolumn{3}{|c|}{ GGA5 } \\
\hline & & $H$ & $F_{1}(\%)$ & $H$ & $F_{1}(\%)$ & $H$ & $F_{1}(\%)$ & $F_{2}(\%)$ & $H$ & $F_{1}(\%)$ & $F_{2}(\%)$ & $H$ & $F_{1}(\%)$ & $F_{2}(\%)$ \\
\hline SM00 & 10 & 80 & 75.26 & 68 & 88.54 & 72 & 83.63 & 85.09 & 71 & 84.8 & 85.88 & 70 & 86.01 & 86.01 \\
\hline SM11 & 11 & 96 & 74.6 & 80 & 89.52 & 88 & 81.38 & 82.41 & 83 & 86.28 & 86.28 & 80 & 89.52 & 89.52 \\
\hline SM12 & 20 & 110 & 70.35 & 90 & 85.98 & 89 & 86.95 & 86.95 & 89 & 86.95 & 86.95 & 87 & 88.94 & 88.94 \\
\hline SM13 & 20 & 113 & 74.5 & 104 & 80.94 & 98 & 85.9 & 87.41 & 95 & 88.61 & 88.61 & 94 & 89.55 & 89.55 \\
\hline SM14 & 31 & 109 & 72.9 & 105 & 75.68 & 91 & 87.32 & 87.32 & 90 & 88.29 & 88.29 & 89 & 89.28 & 89.28 \\
\hline SM15 & 40 & 126 & 73.19 & 118 & 76.23 & 103 & 87.33 & 87.33 & 100 & 89.95 & 89.95 & 97 & 92.73 & 92.73 \\
\hline Average & & & 73.47 & & 82.82 & & 85.42 & 86.09 & & 87.48 & 87.66 & & 89.34 & 89.34 \\
\hline Increase & & & & & 9.35 & & 11.95 & & & 14.01 & & & 15.87 & \\
\hline BM29 & 30 & 106 & 74.47 & 100 & 78.94 & 89 & 88.70 & 89.08 & 89 & 88.70 & 89.91 & 87.4 & 90.32 & 91.56 \\
\hline BM30 & 35 & 96 & 76.92 & 99 & 74.59 & 85 & 86.87 & 87.25 & 85 & 86.87 & 88.01 & 83 & 88.97 & 89.35 \\
\hline BM31 & 40 & 87 & 77.20 & 98 & 68.53 & 78 & 86.11 & 86.48 & 76 & 88.37 & 89.68 & 75 & 89.55 & 89.94 \\
\hline BM32 & 45 & 99 & 77.29 & 102 & 75.02 & 89 & 85.98 & 86.35 & 88 & 86.96 & 87.33 & 88 & 86.96 & 87.33 \\
\hline BM33 & 50 & 88 & 70.71 & 95 & 65.50 & 73 & 85.24 & 85.60 & 71 & 87.64 & 88.02 & 70 & 88.89 & 89.27 \\
\hline BM34 & 55 & 108 & 75.90 & 116 & 70.66 & 96 & 85.38 & 85.75 & 94 & 87.20 & 87.58 & 90 & 91.07 & 91.47 \\
\hline BM35 & 60 & 86 & 76.45 & 95 & 69.21 & 78 & 84.29 & 84.65 & 74 & 88.95 & 90.16 & 73 & 90.06 & 91.39 \\
\hline BM36 & 65 & 91 & 78.27 & 104 & 68.49 & 83 & 85.82 & 86.19 & 82 & 86.87 & 88.00 & 79 & 90.16 & 90.55 \\
\hline BM37 & 70 & 105 & 73.00 & 112 & 68.43 & 89 & 86.12 & 86.49 & 88 & 87.10 & 87.47 & 86 & 89.12 & 89.51 \\
\hline BM38 & 75 & 96 & 79.62 & 107 & 71.44 & 90 & 84.93 & 85.30 & 88 & 86.86 & 88.15 & 86 & 88.88 & 89.27 \\
\hline BM39 & 80 & 107 & 77.03 & 121 & 68.12 & 95 & 86.76 & 87.13 & 93 & 88.63 & 89.93 & 90 & 91.58 & 91.97 \\
\hline Avg29 39 & & & 76.08 & & 70.81 & & 86.02 & 86.39 & & 87.65 & 88.57 & & 89.60 & 90.15 \\
\hline Increase & & & & & -5.27 & & 9.94 & & & 11.57 & & & 13.52 & \\
\hline BM28 & 13 & 77.4 & 67.36 & 61 & 85.47 & 64 & 81.46 & 81.74 & 63.1 & 82.63 & 84.28 & 61 & 85.47 & 88.31 \\
\hline BM40 & 20 & 107.4 & 73.84 & 96 & 82.61 & 92 & 86.20 & 86.50 & 91 & 87.15 & 88.28 & 90 & 88.12 & 88.42 \\
\hline BM41 & 20 & 103.1 & 70.50 & 87 & 83.54 & 85.4 & 85.11 & 85.18 & 85 & 85.51 & 86.41 & 84.1 & 86.42 & 88.01 \\
\hline Average & & & 70.57 & & 83.87 & & 84.26 & 84.47 & & 85.10 & 86.32 & & 86.67 & 88.25 \\
\hline Increase & & & & & 13.31 & & 13.69 & & & 14.53 & & & 16.10 & \\
\hline
\end{tabular}

Table A-3. Comparison of filled rates of the heterogeneous cases by different methods

\begin{tabular}{|c|c|c|c|c|c|c|c|c|c|c|c|c|c|c|}
\hline & & \multicolumn{2}{|c|}{ Rdm } & \multicolumn{2}{c|}{ MILP } & \multicolumn{3}{c|}{ OGA(Swp) } & \multicolumn{3}{c|}{ IGA(Swp) } & \multicolumn{3}{c|}{ GGA5 } \\
\hline & $N$ & $H$ & $F_{1}(\%)$ & $H$ & $F_{1}(\%)$ & $H$ & $F_{1}(\%)$ & $F_{2}(\%)$ & $H$ & $F_{1}(\%)$ & $F_{2}(\%)$ & $H$ & $F_{1}(\%)$ & $F_{2}(\%)$ \\
\hline SM00 & 10 & 80 & 75.26 & 68 & 88.54 & 72 & 83.63 & 85.09 & 71 & 84.8 & 85.88 & 70 & 86.01 & 86.01 \\
\hline SM11 & 11 & 96 & 74.60 & 80 & 89.52 & 88 & 81.38 & 82.41 & 83 & 86.28 & 86.28 & 80 & 89.52 & 89.52 \\
\hline BM28 & 13 & 77.4 & 67.36 & 61 & 85.47 & 64 & 81.46 & 81.74 & 63.1 & 82.63 & 84.28 & 61 & 85.47 & 88.31 \\
\hline BM40 & 20 & 107.4 & 73.84 & 96 & 82.61 & 92 & 86.20 & 86.50 & 91 & 87.15 & 88.28 & 90 & 88.12 & 88.42 \\
\hline BM41 & 20 & 103.1 & 70.50 & 87 & 83.50 & 85.4 & 85.11 & 85.18 & 85 & 85.51 & 86.41 & 84.1 & 86.42 & 88.01 \\
\hline Average & & & $\mathbf{7 2 . 3 1}$ & & $\mathbf{8 5 . 9 3}$ & & $\mathbf{8 3 . 5 6}$ & $\mathbf{8 4 . 1 8}$ & & $\mathbf{8 5 . 2 7}$ & $\mathbf{8 6 . 2 3}$ & & $\mathbf{8 7 . 1 1}$ & $\mathbf{8 8 . 0 5}$ \\
\hline Increase & & & & & $\mathbf{1 3 . 6 2}$ & & $\mathbf{1 1 . 2 4}$ & & & $\mathbf{1 2 . 9 6}$ & & & $\mathbf{1 4 . 8 0}$ & \\
\hline
\end{tabular}


Table A-4. Comparison of computational times of the old cases by different methods

\begin{tabular}{|c|c|c|c|c|c|c|c|c|c|c|c|c|c|c|}
\hline & \multirow[b]{2}{*}{$N$} & \multicolumn{2}{|c|}{ Rdm } & \multicolumn{2}{|c|}{ MILP } & \multicolumn{2}{|c|}{ OGA(Swp) } & \multicolumn{2}{|c|}{ IGA(Swp) } & \multicolumn{3}{|c|}{ GGA5 } & \multicolumn{2}{|c|}{ Comparison } \\
\hline & & $\boldsymbol{H}$ & $C T_{R}$ & $\boldsymbol{H}$ & $C T_{M}$ & $\boldsymbol{H}$ & $C T_{O}$ & $\boldsymbol{H}$ & $C T_{I}$ & $\boldsymbol{H}$ & $C T_{G}$ & $g$ & $C T_{\partial} C T_{I}$ & $C T_{\delta} / C T_{G}$ \\
\hline SM01 & 8 & 92 & 0.031 & $80^{*}$ & 153 & 80 & 11.12 & 80 & 1.25 & 80 & 5.16 & 4 & 8.90 & 2.16 \\
\hline SM02 & 8 & 40 & 0.031 & $37 *$ & 547 & 37 & 14.80 & 37 & 1.56 & 37 & 3.81 & 3 & 9.49 & 3.88 \\
\hline SM03 & 9 & 53 & 0.031 & $47 *$ & 1 & 47 & 15.84 & 47 & 1.39 & 47 & 3.44 & 3 & 11.40 & 4.60 \\
\hline SM04 & 9 & 103 & 0.032 & 82 & 7200 & 83 & 31.85 & 83 & 1.84 & 82 & 6.06 & 3 & 17.31 & 5.26 \\
\hline SM05 & 10 & 61 & 0.046 & $52 *$ & 734 & 52 & 16.36 & 52 & 2.74 & 52 & 6.23 & 3 & 5.97 & 2.63 \\
\hline SM06 & 12 & 48 & 0.047 & $38^{*}$ & 285 & 40 & 29.56 & 40 & 3.02 & 38 & 17.27 & 4 & 9.79 & 1.71 \\
\hline SM07 & 17 & 62 & 0.063 & 62 & 7200 & 62 & 56.48 & 62 & 3.86 & 62 & 9.11 & 3 & 14.63 & 6.20 \\
\hline SM08 & 17 & 77 & 0.063 & 71 & 7200 & 69 & 49.32 & 62 & 7.27 & 62 & 20.27 & 3 & 6.78 & 2.43 \\
\hline SM09 & 19 & 62 & 0.125 & 62 & 7200 & 52 & 79.12 & 51 & 4.95 & 50 & 38.83 & 4 & 15.98 & 2.04 \\
\hline SM10 & 21 & 78 & 0.078 & 62 & 7200 & 63 & 77.88 & 63 & 14.41 & 62 & 51.03 & 5 & 5.40 & 1.53 \\
\hline BM01 & 5 & 84.1 & \begin{tabular}{|l|}
0.031 \\
\end{tabular} & $84.1 *$ & 3 & 84.1 & 24.04 & 84.1 & 1.02 & 84.1 & 2.73 & 3 & 23.57 & 8.81 \\
\hline BM02 & 11 & 99 & 0.062 & 83.1 & 7200 & 84.1 & 96.88 & 84.1 & 5.42 & 84.1 & 14.44 & 3 & 17.87 & 6.71 \\
\hline BM03 & 12 & 47 & 0.109 & $32 *$ & 368 & 32 & 141.76 & 32 & 5.09 & 32 & 17.63 & 3 & 27.85 & 8.04 \\
\hline BM04 & 13 & 98 & 0.078 & 77.4 & 7200 & 83.4 & 108.00 & 77.4 & 10.39 & 77.4 & 37.27 & 3 & 10.39 & 2.90 \\
\hline BM05 & 15 & 90 & 0.094 & 79 & 7200 & 79 & 130.20 & 79 & 18.81 & 78 & 60.75 & 4 & 6.92 & 2.14 \\
\hline BM06 & 16 & 47 & 0.109 & 40 & 7200 & 40 & 253.40 & 40 & 9.36 & 40 & 45.38 & 4 & 27.07 & 5.58 \\
\hline BM07 & 21 & 40 & 0.328 & 40 & 7200 & 40 & 472.36 & 38 & 18.13 & 37 & 72.16 & 4 & 26.05 & 6.55 \\
\hline BM08 & 22 & 54 & 0.734 & 53 & 7200 & 51 & 493.72 & 51 & 16.39 & 48 & 115.55 & 6 & 30.12 & 4.27 \\
\hline BM09 & 23 & 80 & 0.188 & 82 & 7200 & 72 & 408.52 & 71 & 17.83 & 71 & 101.69 & 3 & 22.91 & 4.02 \\
\hline BM10 & 23 & 62 & 0.188 & 63.1 & 7200 & 61 & 475.36 & 61 & 26.20 & 61 & 85.69 & 3 & 18.14 & 5.55 \\
\hline BM11 & 25 & 80.1 & 0.218 & 78 & 7200 & 77.4 & 488.52 & 73.4 & 28.33 & 73.4 & 89.28 & 3 & 17.24 & 5.47 \\
\hline BM12 & 26 & 66 & 0.234 & 68 & 7200 & 61 & 648.08 & 61 & 33.25 & 61 & 89.73 & 3 & 19.49 & 7.22 \\
\hline BM13 & 27 & 85 & 0.250 & 78 & 7200 & 71 & 567.60 & 69 & 39.00 & 68 & 118.69 & 3 & 14.55 & 4.78 \\
\hline BM14 & 28 & 94 & 0.265 & 88.4 & 7200 & 78.4 & 544.92 & 78.4 & 56.30 & 78.4 & 200.02 & 5 & 9.68 & 2.72 \\
\hline BM15 & 29 & 94 & 0.359 & 87 & 7200 & 75 & 660.53 & 74 & 44.72 & 73 & 193.80 & 5 & 14.77 & 3.41 \\
\hline BM16 & 29 & 54 & 0.360 & 52 & 7200 & 48 & 992.68 & 47 & 60.52 & 47 & 174.53 & 3 & 16.40 & 5.69 \\
\hline BM17 & 29 & 101 & 0.265 & 88.4 & 7200 & 80 & 610.40 & 78.4 & 59.19 & 78.4 & 241.58 & 5 & 10.31 & 2.53 \\
\hline BM18 & 30 & 70 & 0.297 & 73.4 & 7200 & 63.1 & 816.08 & 63 & 54.17 & 63 & 130.94 & 3 & 15.07 & 6.23 \\
\hline BM19 & 30 & 78.4 & 0.297 & 79 & 7200 & 63.1 & 800.32 & 63.1 & 66.28 & 63 & 195.64 & 4 & 12.07 & 4.09 \\
\hline BM20 & 30 & 94 & 0.297 & 87 & 7200 & 79 & 657.93 & 79 & 50.89 & 78 & 206.34 & 5 & 12.93 & 3.19 \\
\hline BM21 & 30 & 94 & 0.328 & 87 & 7200 & 78.4 & 661.87 & 78.4 & 50.13 & 78.4 & 141.19 & 3 & 13.20 & 4.69 \\
\hline BM22 & 31 & 85 & 0.313 & 87 & 7200 & 78 & 684.30 & 78 & 62.66 & 77 & 182.74 & 4 & 10.92 & 3.74 \\
\hline BM23 & 33 & 94 & 0.359 & 93.4 & 7200 & 78.4 & 769.54 & 77.4 & 90.92 & 77 & 259.89 & 4 & 8.46 & 2.96 \\
\hline BM24 & 35 & 94 & 0.375 & 89 & 7200 & 80 & 895.13 & 82 & 57.88 & 79 & 492.23 & 9 & 15.47 & 1.82 \\
\hline BM25 & 36 & 87 & 0.422 & 79 & 7200 & 76 & 1071.30 & 73 & 89.08 & 71 & 551.45 & 5 & 12.03 & 1.94 \\
\hline BM26 & 38 & 101 & 0.453 & 97 & 7200 & 82 & 1015.65 & 80 & 106.28 & 80 & 245.70 & 3 & 9.56 & 4.13 \\
\hline BM27 & 40 & 100 & 0.500 & 109 & 7200 & 85 & 1185.35 & 85 & 97.69 & 84 & 334.41 & 4 & 12.13 & 3.54 \\
\hline Average & & & & & & & & & & & & 3.84 & 14.62 & 4.19 \\
\hline
\end{tabular}


Table A-5. Comparison of computational times of the new cases by different methods

\begin{tabular}{|c|c|c|c|c|c|c|c|c|c|c|c|c|c|c|}
\hline & & \multicolumn{2}{|c|}{ Rdm } & \multicolumn{2}{|c|}{ MILP } & \multicolumn{2}{|c|}{ OGA(Swp) } & \multicolumn{2}{|c|}{ IGA(Swp) } & \multicolumn{3}{|c|}{ GGA5 } & \multicolumn{2}{|c|}{ Comparison } \\
\hline & $N$ & $H$ & $C T_{R}$ & $H$ & $C T_{M}$ & $H$ & $C T_{O}$ & $H$ & $C T_{I}$ & $H$ & $C T_{G}$ & $g$ & $C T_{d} C T_{I}$ & $C T_{\delta} / C T_{G}$ \\
\hline SM00 & 10 & 80 & 0.031 & $68^{*}$ & 1144 & 72 & 22.19 & 71 & 2.47 & 70 & 7.20 & 4 & 8.98 & 3.08 \\
\hline SM11 & 11 & 96 & 0.031 & 80 & 7200 & 88 & 18.50 & 83 & 2.69 & 80 & 9.31 & 5 & 6.88 & 1.99 \\
\hline SM12 & 20 & 110 & 0.062 & 90 & 7200 & 89 & 96.79 & 89 & 11.79 & 87 & 30.38 & 4 & 8.21 & 3.19 \\
\hline SM13 & 20 & 113 & 0.063 & 104 & 7200 & 98 & 141.32 & 95 & 8.99 & 94 & 25.55 & 4 & 15.72 & 5.53 \\
\hline SM14 & 31 & 109 & 0.188 & 105 & 7200 & 91 & 264.47 & 90 & 22.06 & 89 & 111.38 & 4 & 11.99 & 2.37 \\
\hline SM15 & 40 & 126 & 0.235 & 118 & 7200 & 103 & 742.48 & 100 & 49.42 & 97 & 225.91 & 7 & 15.02 & 3.29 \\
\hline BM28 & 13 & 77.4 & 0.093 & 61 & 7200 & 64 & 126.49 & 63.1 & 9.45 & 61 & 43.13 & 5 & 13.39 & 2.93 \\
\hline BM29 & 30 & 106 & 0.328 & 100 & 7200 & 89 & 1199.79 & 89 & 57.66 & 87.4 & 237.34 & 5 & 20.81 & 5.06 \\
\hline BM30 & 35 & 96 & 0.391 & 99 & 7200 & 85 & 856.47 & 85 & 72.56 & 83 & 285.92 & 5 & 11.80 & 3.00 \\
\hline BM31 & 40 & 87 & 0.500 & 98 & 7200 & 78 & 1278.47 & 76 & 101.77 & 75 & 424.41 & 5 & 12.56 & 3.01 \\
\hline BM32 & 45 & 99 & 0.625 & 102 & 7200 & 89 & 2602.36 & 88 & 151.75 & 88 & 600.91 & 4 & 17.15 & 4.33 \\
\hline BM33 & 50 & 88 & 0.797 & 95 & 7200 & 73 & 2607.48 & 71 & 215.23 & 70 & 1016.83 & 5 & 12.11 & 2.56 \\
\hline BM34 & 55 & 108 & 0.984 & 116 & 7200 & 96 & 4717.27 & 94 & 235.84 & 90 & 802.78 & 4 & 20.00 & 5.88 \\
\hline BM35 & 60 & 86 & 1.297 & 95 & 7200 & 78 & 4326.64 & 74 & 318.31 & 73 & 1057.34 & 4 & 13.59 & 4.09 \\
\hline BM36 & 65 & 91 & 1.500 & 104 & 7200 & 83 & 4689.35 & 82 & 409.11 & 79 & 1380.56 & 3 & 11.46 & 3.40 \\
\hline BM37 & 70 & 105 & 1.797 & 112 & 7200 & 89 & 8436.69 & 88 & 605.63 & 86 & 1868.84 & 5 & 13.93 & 4.51 \\
\hline BM38 & 75 & 96 & 2.140 & 107 & 7200 & 90 & 11188.73 & 88 & 600.88 & 86 & 2014.80 & 4 & 18.62 & 5.55 \\
\hline BM39 & 80 & 107 & 2.500 & 121 & 7200 & 95 & 14601.00 & 93 & 741.09 & 90 & 2015.97 & 4 & 19.70 & 7.24 \\
\hline BM40 & 20 & 107.4 & 0.141 & 96 & 7200 & 92 & 488.04 & 91 & 23.08 & 90 & 62.31 & 4 & 21.15 & 7.83 \\
\hline BM41 & 20 & 103.1 & 0.140 & 87 & 7200 & 85.4 & 248.97 & 85 & 22.52 & 84.1 & 97.41 & 4 & 11.06 & 2.56 \\
\hline Average & & & & & & & & & & & & 4.45 & 14.21 & 4.07 \\
\hline
\end{tabular}

